\title{
Systematic Combination of Oligonucleotides and Synthetic Polymers for Advanced Therapeutic Applications
}

\author{
Moohyun Han ${ }^{\dagger, 1}$ \\ Jiyun Beon ${ }^{\dagger, 1}$ \\ Ju Young Lee ${ }^{2}$ \\ Seung Soo $\mathrm{Oh}^{*, 1}$
}

\author{
${ }^{1}$ Department of Materials Science and Engineering, Pohang University of Science Technology (POS- \\ TECH), Pohang, Gyeongbuk 37673, Korea \\ ${ }^{2}$ Research Center for Bio-based Chemistry, Korea Research Institute of Chemical Technology \\ (KRICT), Ulsan 44429, Korea
}

Received June 30, 2021 / Revised August 22, 2021 / Accepted September 25, 2021

\begin{abstract}
The potential of oligonucleotides is exceptional in therapeutics because of their high safety, potency, and specificity compared to conventional therapeutic agents. However, many obstacles, such as low in vivo stability and poor cellular uptake, have hampered their clinical success. Use of polymeric carriers can be an effective approach for overcoming the biological barriers and thereby maximizing the therapeutic efficacy of the oligonucleotides due to the availability of highly tunable synthesis and functional modification of various polymers. As loaded in the polymeric carriers, the therapeutic oligonucleotides, such as antisense oligonucleotides, small interfering RNAs, microRNAs, and even messenger RNAs, become nuclease-resistant by bypassing renal filtration and can be efficiently internalized into disease cells. In this review, we introduced a variety of systematic combinations between the therapeutic oligonucleotides and the synthetic polymers, including the uses of highly functionalized polymers responding to a wide range of endogenous and exogenous stimuli for spatiotemporal control of oligonucleotide release. We also presented intriguing characteristics of oligonucleotides suitable for targeted therapy and immunotherapy, which can be fully supported by versatile polymeric carriers.

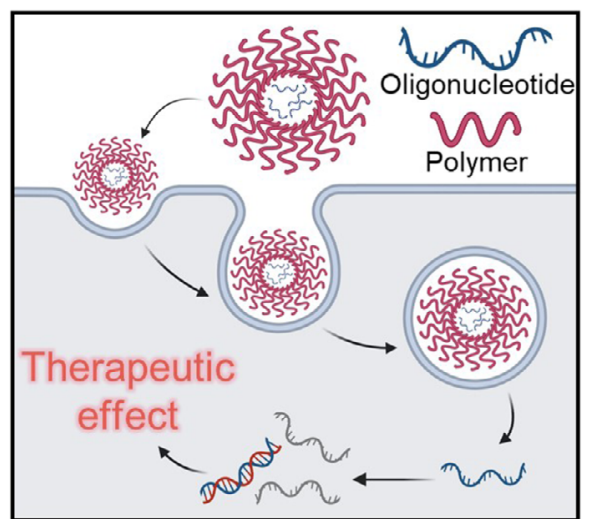

Keywords: nucleic acid, gene delivery, polymeric carrier, stimuli-responsive system, immunotherapy.

\section{Introduction}

Health is one of the most important things in our lives. Currently, we suffer from the global pandemic, COVID-19 caused by severe acute respiratory syndrome coronavirus $2,{ }^{1}$ and as our lives can be frequently threatened by various types of diseases, including infectious diseases, genetic disorders, and even degenerative diseases, developments of their appropriate treatments have attracted a great deal of attention up to date, and extensive studies are still ongoing. ${ }^{2-4}$ In particular, oligonucleotide therapeutics, which employs chemically synthesizable oligonucleotides such as deoxyribonucleic acids (DNAs) and ribonucleic acids (RNAs) as potent therapeutic agents, has proven its clinical potential in treating various diseases since the first report in $1978 .{ }^{5}$ On the basis of the central dogma of molecular biology, the mechanism of action for this oligonucleotide-based therapy involves in controlling cellular signals by targeting specific messenger RNAs (mRNAs) that are translated into the proteins with pathological effects; compared to conventional therapies relying on small molecules, peptides, and proteins, the oligonu-

Acknowledgment: This work was supported by the National Research Foundation of Korea (NRF) grant [NRF-2017R1C1B3012050], and figures were prepared using BioRender.Com for scientific illustrations.

*Corresponding Author: Seung Soo Oh (seungsoo@postech.ac.kr)

${ }^{\dagger}$ These authors equally contributed to this work. cleotide therapy enabling selective regulation of gene expression demonstrates greater safety, stronger potency, and higher specificity, along with an unrestricted choice of drug targets. ${ }^{6}$

Even though various therapeutic oligonucleotides have opened a new era of disease treatments through their novel and efficient mechanisms, several problems, including low in vivo stability and fast renal clearance of the oligonucleotides, still remain. ${ }^{7,8}$ To address these problems, there have been many studies on the development of oligonucleotide carriers using a variety of organic and inorganic materials. ${ }^{9,10}$ Among them, lipid-based carriers, including liposomes, have been clinically used for oligonucleotide delivery as improving tissue penetration. ${ }^{11}$ Allowing tunable surface modification, inorganic or metal nanoparticles have shown a long-term stability and a photothermal effect, all of which are highly advantageous for therapeutic applications of oligonucleotides. ${ }^{12}$ Even spherical forms of nucleic acids have been used as nanocarriers of therapeutic oligonucleotides; as designed in a highly oriented manner and recognize other target nucleic acids, the spherical nucleic acids have been also applied in theranostics. $^{13}$

By effective collaboration with functional synthetic polymers, the oligonucleotide-based therapy can be more powerful. ${ }^{14-16}$ Polymer-based nanoparticles can secure the loaded oligonucleotides during in vivo circulation, and simultaneously enhance cellular uptake; for example, polyethylene glycol (PEG) and polyethylenimine (PEI) have proven their effectiveness in avoid- 
ing renal filtration and interacting with cell membranes for the maximal therapeutic effect of oligonucleotides. Compared to other material types of carriers, various polymers (e.g., poly(lactic acid) (PLA) and poly(lactic-co-glycolic acid) (PLGA)) can be prepared to be biodegradable and biocompatible for in vivo applications, and the availability of highly tunable, chemical synthesis and functional modifications allows the resulting polymers to perform programmed functions capable of significantly improving the therapeutic effects of oligonucleotides. ${ }^{17}$ Elaborate construction of polymeric carriers is also achievable; surface charge and size of nanocarriers can be controlled for therapeutic purposes, ${ }^{18,19}$ and within dendrimers and core-shell structured polymers, inorganic and metal nanomaterials are encapsulated for further employing their diagnostic and therapeutic functions. ${ }^{20,21}$ Taken together, the versatile polymers could be regarded not as simple drug carriers, but as effective treatment boosters.

In this review, we presented how oligonucleotides and synthetic polymers can be synergistically combined for therapeutic applications. First of all, we discussed the distinct advantages of the oligonucleotide-polymer combination in improving therapeutic efficacy and efficiency; to guide specific gene-recognizing oligonucleotides to be delivered into disease cells, the polymeric carriers with cell penetration capabilities secure the loaded oligonucleotides against nuclease degradation, simultaneously prolonging their circulation time. In terms of unique characteristics of the therapeutic oligonucleotides, we also introduced what kinds of synthetic polymers have been explored for effective oligonucleotide delivery, exemplifying a wide range of stimuliresponsive polymeric systems. Furthermore, we demonstrated that newly emerging functions of oligonucleotides, such as molecular recognition and immune response induction, can benefit the applications of the polymeric drug carriers. Finally, we briefly discussed the challenges in clinically using the combination of functional oligonucleotides and polymers toward creation of the next-generation therapeutic applications.

\section{Advantages of combining oligonucleotides and functional polymers}

Strategic combination between therapeutic oligonucleotides and various polymers is highly valuable for maximizing the therapeutic efficacy in oligonucleotide therapy (Figure 1). Based on
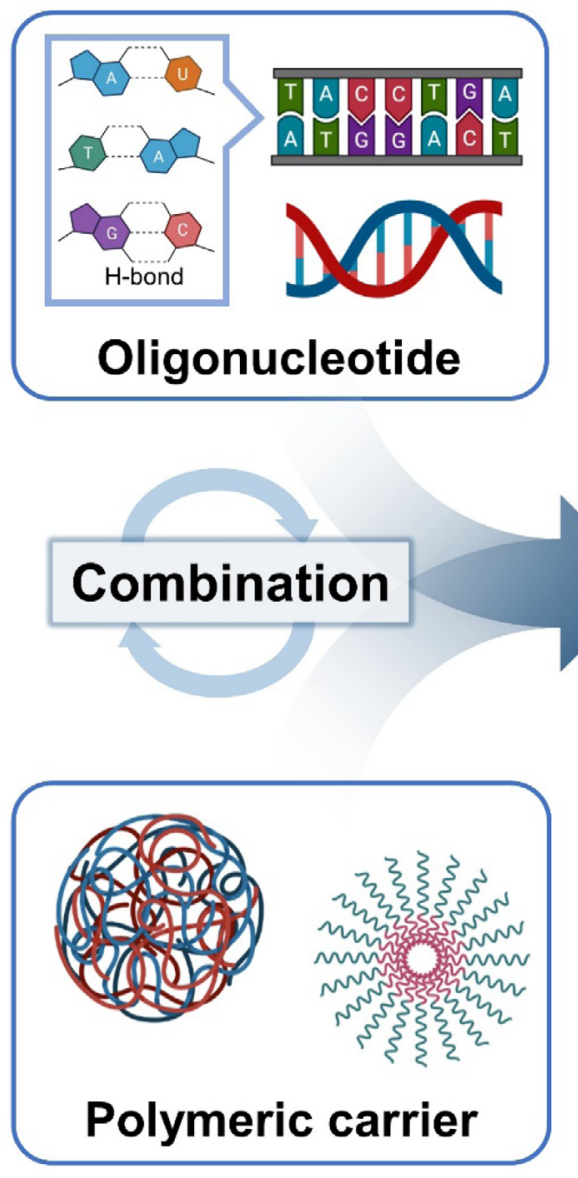

Figure 1. Many advantages of combining oligonucleotides with synthetic polymers for therapeutic applications. Specifically designed oligonucleotides can be used to inhibit disease-related genes or express therapeutic proteins in our bodies. Various polymeric carriers can be used to improve the therapeutic efficacy and efficiency of the oligonucleotides; by preventing nuclease degradation and renal filtration, the in vivo stability and blood circulation time of the oligonucleotides can be further enhanced, while the availability of co-drug delivery makes synergistic oligonucleotide therapy achievable. Moreover, the cellular uptake of the oligonucleotides can be promoted by the polymeric carriers, and in particular, the stimuli-responsive polymers enable the localization of oligonucleotide release into disease sites for minimizing side effects. The valuable polymeric carriers can be multifunctionalized to perform not only the oligonucleotide delivery, but also disease diagnosis and in vivo imaging for advanced theranostics. 
the flow of genetic information in a biological system from DNA to RNA to protein, regulation of the transient RNAs can be an effective approach capable of modulating the levels of proteins relevant to various diseases. ${ }^{22}$ To date, there have been several gene regulation mechanisms mediated by therapeutic oligonucleotides, such as antisense oligonucleotides (ASOs), ${ }^{23}$ small interfering RNAs (siRNAs), ${ }^{24}$ and microRNAs (miRNAs), ${ }^{25}$ and their therapeutic effects have been validated by a number of scientific reports and clinical studies. ${ }^{26}$ In addition to the therapeutic functions, some oligonucleotides, which can form specific 3D conformations at specific $\mathrm{pH}$ and ionic conditions, even perform molecular recognition like antibodies (e.g., aptamers) ${ }^{27-29}$ and catalytic reactions like enzymes (e.g., DNAzymes and ribozymes). ${ }^{30,31}$ Based on dynamic DNA structures (e.g., i-motif and azobenzene-modified DNA), oligonucleotides themselves serve as smart nanocarriers. ${ }^{32}$ These oligonucleotides, however, have exposed inherent limitations in therapeutically applying them for in vivo systems, which can be readily addressed by use of polymeric carriers. ${ }^{26}$ Compared to viral carriers, the polymeric oligonucleotide carriers have many different advantages including a large capacity in oligonucleotide delivery, structural flexibility in carrier design, and even biological safety in clinical use. ${ }^{33}$ Furthermore, to respond to unique conditions around abnormal cells, such as a tumor microenvironment, ${ }^{34,35}$ they can be functionalized to selectively release the delivered oligonucleotides at specific sites.

\subsection{Specific gene targeting}

Using Watson-Crick base-pairing interactions, ${ }^{36}$ oligonucleotides can recognize disease-relevant specific genes, thereby exhibiting strong therapeutic effects. As comprising a string of nucleotides including adenine, guanine, cytosine, and thymine (or uracil), the therapeutic oligonucleotides can be complementary to target genes as held together by hydrogen bonding. For therapeutic applications, the oligonucleotides are typically designed to bind or degrade specific mRNAs, which are destined not to interact with ribosomes, thereby inhibiting a subsequent translation process. By silencing mRNAs, there can be no expression of disease-relevant proteins, alleviating the symptoms of the disease. $^{37}$

\subsection{Enhancing in vivo stability}

Oligonucleotides are unstable in our bodies because of their rapid degradation by ubiquitous nucleases. To assess their in vivo stability, the viability of RNAs was tested in clinical samples, and as a result, the concentration of endogenous RNAs decreased by more than $99 \%$ in blood, serum, and plasma within 15 seconds. ${ }^{38}$ Even though DNAs are known to be more stable than the RNAs, the DNA administration into a mouse showed that the degradation time of intravenously injected naked plasmid DNAs (pDNAs) was only 15 minutes. ${ }^{39}$ In addressing this low in vivo stability of oligonucleotides, the complex formation between the oligonucleotides and biocompatible polymers have been useful to avoid unwanted interactions with various blood components including nucleases. In a study, cat- ionic polymers carrying negatively charged oligonucleotides have shown more than $650 \%$ increase in the degradation time of the oligonucleotides by blocking the accessibility of nucleases. $^{40}$

\subsection{Prolonging blood circulation time}

Renal filtration is a size-dependent removal process of metabolic waste products (e.g., urea and creatinine), but it can also cause therapeutic agents with a small size and low molecular weight, including oligonucleotides, to be eliminated from our bodies, resulting in significant reduction of therapeutic efficiency (Figure 2(A)). Use of polymeric carriers can be a great way to bypass the renal filtration by increasing the total size of the complex; it can improve the therapeutic effect of the oligonucleotides by prolonging their circulation time in bloodstream. Previously, polyethylene glycol (PEG) was conjugated with small interfering locked nucleic acids (siLNAs) to prolong the blood circulation time of the oligonucleotides and prevent their urine excretion. ${ }^{8}$ To exclude the effect of enzymatic degradation, the siLNAs (i.e., the modified RNAs with enzymatic resistance) were used instead of common siRNAs. When the naked siLNAs and the siLNAs conjugated with $20 \mathrm{kDa}$ PEGs (PEG20k-siLNAs) were compared each other, the naked siLNAs were degraded over $90 \%$ within 15 minutes, but the PEG20k-siLNAs remained $50 \%$ and $25 \%$ after $1 \mathrm{~h}$ and $2 \mathrm{~h}$, respectively. This result demonstrated that the PEGylated oligonucleotides can effectively avoid the renal filtration, thereby lengthening their circulation time and half-life in the blood stream.

\subsection{Cell internalization}

The polyplex formation between negatively charged oligonucleotides and cationic polymers is highly effective to improve the in vivo stability of the oligonucleotides and simultaneously, their internalization efficiency into cells (Figure 2(B)). ${ }^{23,41}$ Similar with the oligonucleotides, the lipid membranes of the cells are charged negatively, so the repulsive charge interaction makes the cell internalization of the oligonucleotides significantly challenging. From this electrostatic point of view, cationic polymers can not only neutralize the negative charge of oligonucleotides, but also exhibit an overall positive zeta potential which can strongly interact with cell membranes; as non-specific endocytosis is promoted, the cell internalization rate of oligonucleotides can increase. Moreover, the polymeric carriers can be further protonated within the endosome by the acidic environment, and due to the proton sponge effect that facilitates the influx of counterions and water molecules at the endosomal $\mathrm{pH}$, the membrane of the endosome can be disrupted; ${ }^{42}$ consequently, the endocytosed oligonucleotides can readily escape from the endosome, leading to the improvement of their therapeutic efficiency.

\subsection{Co-drug delivery}

Co-delivery systems are effective for immunotherapy and cancer therapy by promoting a synergistic effect of therapeutic oligonucleotides; ${ }^{43-45}$ specific design of block copolymers allows 
the resulting polymeric carriers to carry multiple types of drugs, regardless of their hydrophobicity and hydrophilicity. Many studies have reported that chemotherapy drugs have a greater effect on cancer therapy when combined with oligonucleotide therapy. ${ }^{46}$ Similarly, the therapeutic effect of immunotherapy is also known to be magnified by delivering immune-inducing antigens and immune-enhancing $\mathrm{CpG}$ oligodeoxynucleotides together. ${ }^{47}$ In the co-delivery system, block copolymers can be synthesized for well-defined structures suitable for loading all the required therapeutic agents. For example, as amphiphilic block copolymers can form micelles, hydrophobic drugs are condensed into the hydrophobic inner core, and negatively charged oligonucleotides are loaded onto the cationic shell. ${ }^{48,49}$

\subsection{Responding to specific stimuli}

Polymeric carriers can be smart enough to perform spatiotemporal control of loaded oligonucleotides in response to various stimuli, such as $\mathrm{pH}$, temperature, and even levels of reactive oxygen species (ROS) ${ }^{50,51}$ When the oligonucleotide-loaded polymers arrive at disease sites, the therapeutic oligonucleotides should be selectively unloaded to exclude side effects (e.g., killing normal cells). For example, as the $\mathrm{pH}$ around tumors (6.6 6.8) is lower than physiological $\mathrm{pH}(\sim 7.4)$, $\mathrm{pH}$-responsive polymeric carriers can be highly useful to localize the release of therapeutic oligonucleotides around the tumors. ${ }^{52}$ Moreover, the polyplexes are typically internalized into the cells through nonspecific endocytosis, so their endosomal escape should be followed to prevent lysosomal degradation of the oligonucleotides. In this regard, synthetic polymers can be designed to increase the transfection efficiency of delivered oligonucleotides into target cells; by responding to environment-specific stimuli, they can selectively release the therapeutic oligonucleotides at specific locations, such as tumors and inflammation cells, thereby maximizing the therapeutic efficiency of oligonucleotide therapy.

\subsection{Multifunctionalization}

Some oligonucleotides are capable of recognizing biological molecules, such as proteins and metabolites, and even performing enzymatic reactions, ${ }^{53-55}$ so the capability of polymeric carriers can be further enhanced and multiplexed as coupled with such functional oligonucleotides. Even though the oligonucleotides can be chemically produced by solid-phase synthesis, the sequencedefined structures can fold into unique conformations at physiological environments; like monoclonal antibodies or membrane receptors, aptamers selectively bind to target molecules, ${ }^{56}$ and like biological enzymes, nucleozymes conduct biocatalytic reactions. ${ }^{28}$ For instance, some aptamers are capable of specifically recognizing cancer cells and have been used as active targeting agents for various drug delivery systems.$^{57}$ As the biological functions cannot be achieved by typical polymers, so systematic combination of functional oligonucleotides and polymers can expand the versatility of the polyplex in therapeutic applications. ${ }^{58,59}$

\section{Polymeric carrier-based oligonucleotide delivery}

Even though the potential of oligonucleotide therapeutics has been widely investigated by exploring the unique characteristics of oligonucleotides (e.g., specific gene recognition and induction of gene silencing), the negatively charged and vulnerable oligonucleotides are susceptible to decomposition by enzymes in our bodies, causing the low efficiency of cellular uptake. ${ }^{11,60}$ By employing functionalized polymers, we can compensate for the drawbacks of the therapeutic oligonucleotides; ${ }^{61}$ for example, the polymers with a large hydrodynamic volume (e.g., PEGs) protect them from enzymatic digestion and reduce their immunogenicity, while cationic polymers (e.g., polyethylenimine (PEI)) can improve the cellular uptake efficiency of the oligonucleotides by electrostatically interacting with negatively charged cell membranes (Figure 2). ${ }^{23}$ In this chapter, we introduced various therapeutic oligonucleotides that can be readily loaded into polymeric carriers and subsequently delivered inside the cells. To maximize the therapeutic efficacy of the oligonucleotides, a broad range of smart polymeric systems that can respond to various stimuli have been developed to date, so we also introduced their applications for advanced oligonucleotide drug delivery.

\subsection{Therapeutic oligonucleotides as cargo molecules}

Oligonucleotides can perform various therapeutic functions, such as gene inhibition, gene activation, vaccination, and even gene editing, through modulating gene expression. Typically, the oligonucleotide therapeutics relies on several forms of oligonucleotides, including ASO, siRNA, miRNA, and mRNA (Figure 3). Whereas ASO, siRNA, and miRNA are delivered into the cytoplasm to target specific mRNA for gene expression inhibition, mRNA as a therapeutic agent induces gene expression as delivered inside the cells. ${ }^{62,63}$ Their structural differences are distinct; for instance, ASO is a form of a single strand, but siRNA and miRNA hold a duplex structure with a short length, while mRNA is a much longer single strand. In consideration of these differences, a variety of polymeric carriers have been extensively studied. ${ }^{64}$

\subsubsection{ASO}

The first ASO therapy was reported in the late 1970s, and since then, a number of studies on the ASO therapy have been demonstrated. ${ }^{65,66}$ As the ASO structure is short and single-stranded, the chemical modification of ASOs is relatively easy, taking advantage of the advances in nucleotide chemistry. For instance, introduction of chemical modifications, such as phosphorothioate backbones, 2'-0-methoxyethyl ribose sugars, and NAcetylgalactosamine, into the ASOs led them to be less vulnerable to nucleases and more probable to be endocytosed by cells. ${ }^{67}$ However, it cannot be sufficient for the in vivo use of the therapeutic ASOs; along with the chemical modifications, the combination of the ASOs with polymeric carriers has been considered to protect the loaded ASOs for successfully inhibiting target genes within the cells. ${ }^{68,69}$ In particular, polymeric nanoparticles can be positively charged to enhance the cellular uptake of ASOs, ${ }^{70}$ and by controlling the degradation rate of the polymeric 


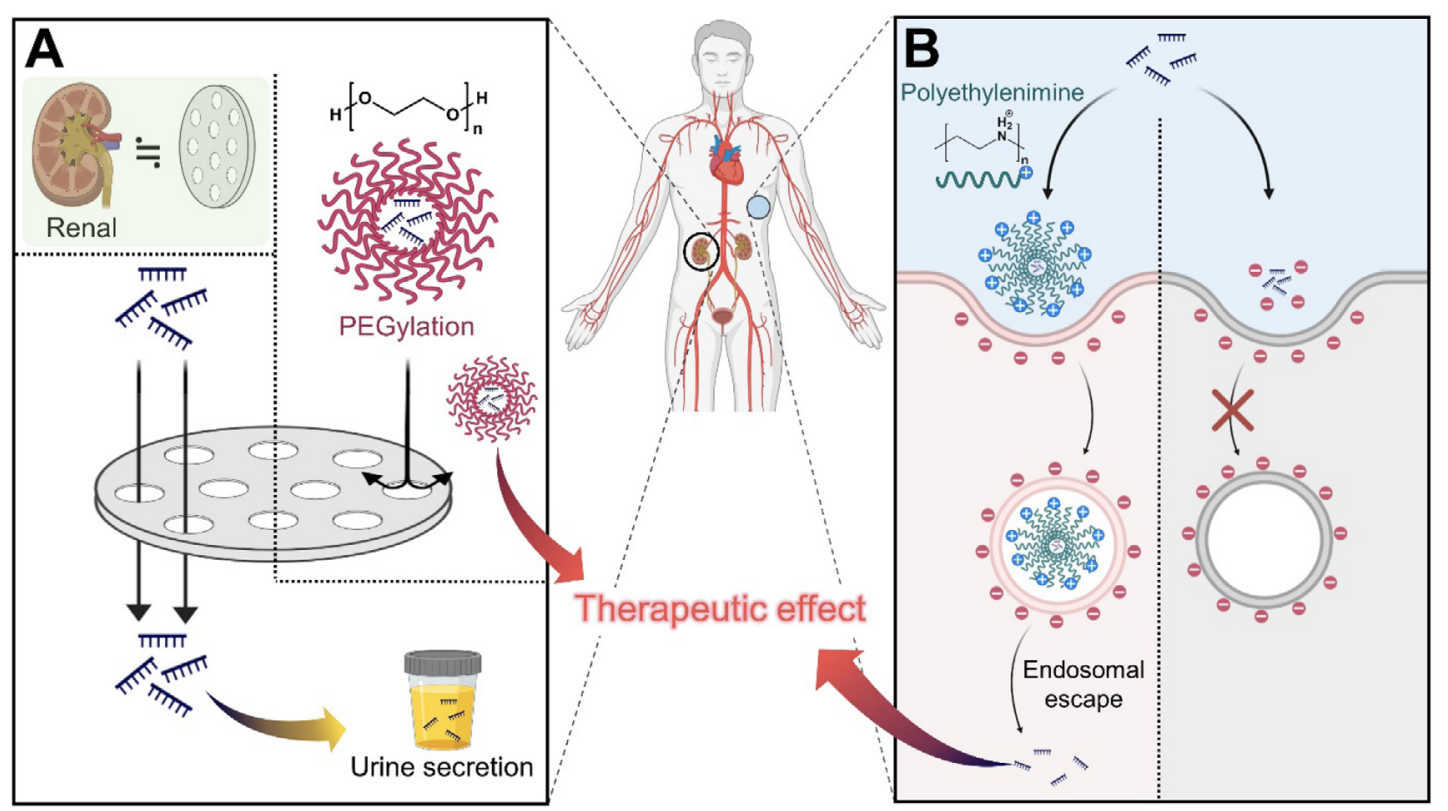

Figure 2. Polymeric strategies to overcome the barriers of in vivo oligonucleotide delivery. (A) Oligonucleotide PEGylation to avoid renal filtration. Due to the small size, oligonucleotides are rapidly cleared by the renal filtration process, leading to urine secretion. By PEGylation, the hydrodynamic volume of the oligonucleotides can be enlarged, thereby prolonging their circulation time in blood. (B) Use of cationic polymers to promote cellular uptake. As both oligonucleotides and cell membranes are negatively charged, electrostatically repulsive interactions occur, making the oligonucleotides difficult to be internalized into cells. Cationic polymers, such as polyethylenimine, can be complexed with the anionic oligonucleotides, making the net charge of the resulting polyplex advantageous for the following cellular uptake. Once internalized into the endosome, the polymeric carriers begin to be protonated at the endosomal $\mathrm{pH}$, causing the membrane disruption by the influx of counterions and water molecules. As resulted by the proton sponge effect, the therapeutic oligonucleotides escape from the endosome and diffuse into the cytoplasm.

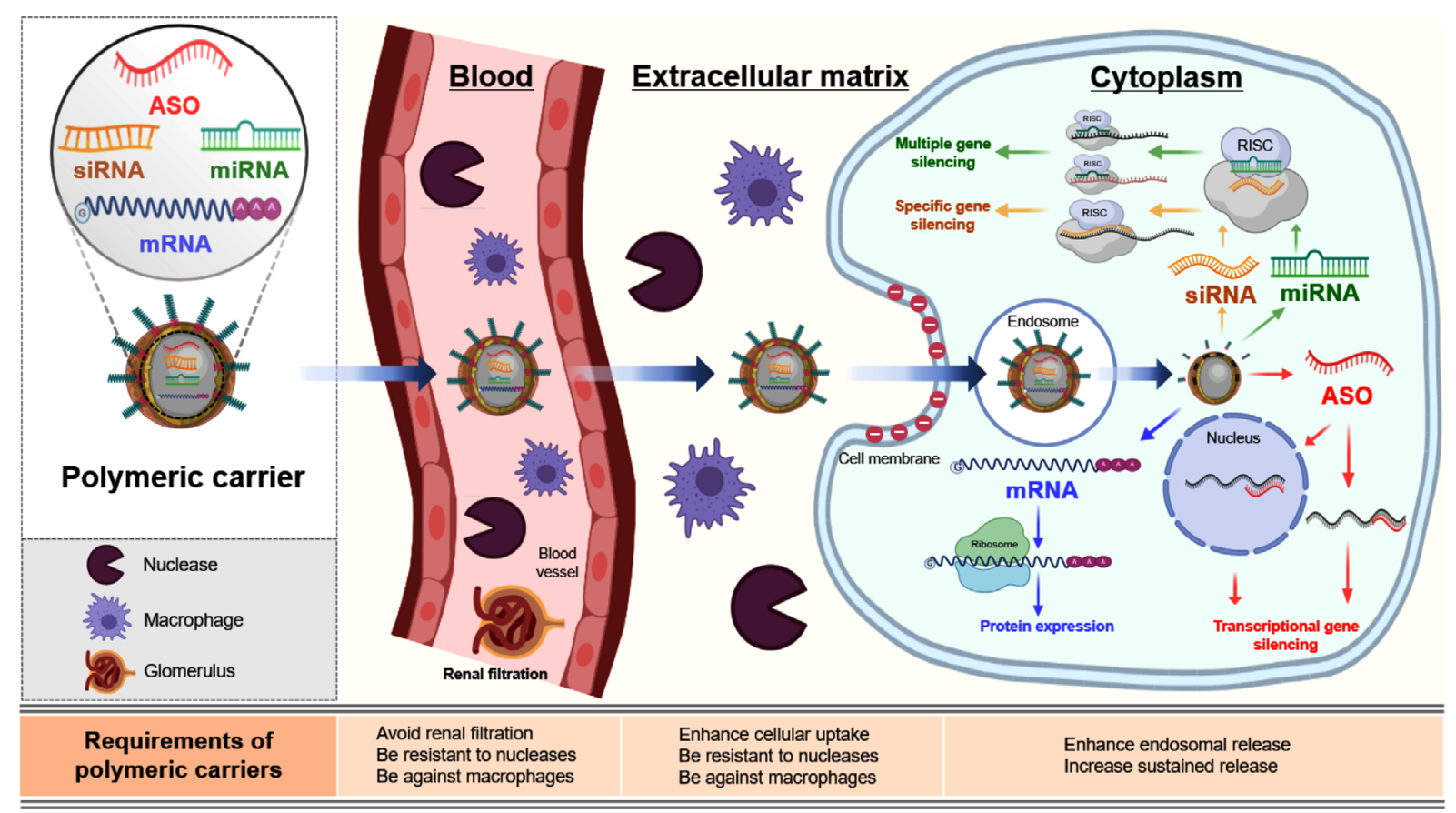

Figure 3. Therapeutic mechanisms of several oligonucleotides and the roles of polymeric carriers for successful oligonucleotide therapy. Polymeric carriers can encapsulate various therapeutic oligonucleotides, such as ASOs, siRNAs, miRNAs, and mRNAs, preventing the nucleolytic degradation by nucleases and macrophages in blood, while the enlarged size of the polyplexes was effective to avoid renal filtration. When the oligonucleotide-loaded polymers successfully reach disease cells by passing through extracellular matrix, the anionic oligonucleotides neutralized by the cationic polymers are well accepted by negatively charged cell membranes, promoting cellular uptakes. When the various oligonucleotides are internalized into the cytoplasm of disease cells, their therapeutic mechanisms are quite different. ASOs can directly bind to pre-mRNAs in nucleus or to mRNAs in cytosol in inhibiting target genes. siRNAs and miRNAs are required to form RNA-induced silencing complexes (RISCs) in cytosol, and the RISCs degrade target mRNAs to inhibit further translation; while siRNAs are specific to bind one target gene, less specific miRNAs enable recognition of multiple mRNAs, leading to multiple gene silencing. mRNAs are decoded by ribosome in cytoplasm, inducing protein expression for vaccination and therapy. 
carriers, sustained release of ASOs has been achievable. ${ }^{71}$

Cationic polymers have been frequently used to decrease the electrostatic repulsive force between ASOs and cell membranes, thereby improving the ASOs' cellular uptake. ${ }^{70}$ Amine-rich PEI has a high density of positive charge, so it can be well combined with the negatively charged ASOs by electrostatic interactions. Because the net charge of ASO/PEI complex can become neutral, the electrostatic repulsion against the negatively charged cell membranes can be cancelled. Moreover, as the PEI can load lots of negatively charged oligonucleotides, including the ASOs, there have been many studies that utilized the cationic PEI as an oligonucleotide drug carrier. ${ }^{23,72}$ In one study, the PEI/ASO complex was delivered into the human bladder carcinoma cell (T24) for inhibition of Ha-ras mRNA, one of the oncogenes. ${ }^{73}$ Compared to the native ASOs without the PEI carrier, the PEI/ ASO complex increased cellular uptakes by $750 \%$, confirming the usefulness of the PEI in neutralizing the negative charge of ASOs to improve the cellular uptake efficiency.

Importantly, ASOs are highly susceptible to nucleases, so they can be rapidly degraded in serum. This means that the therapeutic ASOs are necessary to be protected by nuclease-resistant carriers, which is effective to maintain the concentrations of the ASOs above a certain level for high therapeutic efficacy. ${ }^{74}$ Accordingly, many studies have been conducted to develop polymeric carriers which can protect the ASOs from nucleases and simultaneously release them in a sustained manner. To this end, biodegradable polymers have often been studied, ${ }^{75}$ and among them, poly(lactic-co-glycolic acid) (PLGA) has been widely used because this polymer is degraded into lactic acids and glycolic acids by esterase in our bodies and eventually decomposed into carbon dioxide and water through the Krebs cycle. ${ }^{76}$ Even after phosphodiester modification, the ASOs were reported to be completely decomposed in serum within an hour, but the ASOs combined with the PLGA retained their full length over 21 days, enabling a sustainable release with more than a 500-fold enhancement in in vivo stability. $^{77}$

\subsubsection{SiRNA}

Compared to other therapeutic oligonucleotides, siRNA would be the most specific for gene silencing. ${ }^{78,79}$ It can effectively downregulate the expression of undesired gene by explicitly recognizing target mRNA, and its gene silencing effect is more reproducible than that of $\mathrm{ASO}^{80}$ When we know the sequence of the target gene, the gene-specific siRNA can be easily designed and synthesized, so the siRNA therapy has attracted the strong attention for treating various diseases, ranging from cancers to even chronic and genetic disorders. ${ }^{79}$ However, similar with ASOs, poor cellular uptake and nucleolytic degradation by nucleases hampers the efficacy of the therapeutic siRNA, ${ }^{79}$ and importantly, the siRNA is easily cleared by kidney due to its small size. ${ }^{63}$ To overcome these critical barriers, the siRNA inevitably requires a carrier such as a polymeric nanoparticle.

PEGylation is an effective way to enhance in vivo stability of siRNA, which is vulnerable to degradation by nucleases and macrophages in the body. The siRNA has a well-defined structure as a double-stranded RNA, and its length is quite short (19 to $23 \mathrm{bp}$ ), yielding low molecular weight $(13 \sim 15 \mathrm{kDa}) .{ }^{81}$ Because of the small volume, the siRNA is easily removed from the body by kidney filtration. Considering the pore size of the glomerular filtration barrier is $8 \mathrm{~nm}$, the siRNA carrier should be enlarged up to $20 \mathrm{~nm}$ in diameter. ${ }^{82}$ The PEGylation can make the siRNA resist against nucleases and opsonins by steric hindrance, because highly flexible PEG chains can coordinate many water molecules to increase the hydrodynamic volume of the PEGylated siRNA. ${ }^{83}$ For example, while naked $25 \mathrm{kDa}$ PEI protected only $9 \%$ of siRNA from RNases, the PEIs with 2, 5, and $20 \mathrm{kDa}$ PEGs prevented the decomposition of siRNA more efficiently, and after $0.5 \mathrm{hr}, 32 \%$, $68 \%$, and $92 \%$ of the loaded siRNAs remained, respectively. ${ }^{84}$ This result showed that PEGylation can provide the sterically shielding effect to siRNAs and the shielding efficiency depends on the length of PEG chains.

Low transfection efficiency of siRNAs is one of the biggest problems in siRNA delivery, and copolymers with cleavable bonds can enhance endosomal release of the oligonucleotides, thereby promoting their transfection into cells. By PEGylation, the volume of polymeric nanoparticles can become sufficiently large to avoid renal filtration and prevent non-specific interactions with opsonin. However, the PEGylation lowers the specificity of plasma membranes, resulting in a reduction of cellular uptake and endosomal escape. ${ }^{85}$ Even though the cellular uptake can be overcome to some extent with the help of membrane receptor ligands, the use of the ligands is insufficient to address the inefficient endosomal release of siRNAs. As the gene regulation process occurs in cytoplasm, the siRNA should be released from the endosome to form the RNA-Induced Silencing Complex (RISC). ${ }^{79}$ To improve the endosomal release of siRNAs, reducible poly(triethylenetetramine/cystamine bisacrylamide) (poly(TETA/ CBA)) nanoparticles were developed ${ }^{86}$ Disulfide linkages in CBA were cleaved by glutathione (GSH), which is abundant in the cytoplasm, so as liberated from the polymeric carriers, the siRNAs were encouraged to release for the endosome. In this study, the use of poly(TETA/CBA) carrying siRNAs resulted in twice less expression of vascular endothelial growth factor (VEGF) than that of linear PEI in human prostate cancer cells (PC-3). This effective suppression of VEGF was caused by GSH-responsive cleavage of disulfide bonds within the polymeric carriers; when buthionine sulfoximine, a GSH inhibitor, was present, there was no difference in the level of VEGF expression, regardless of the presence of poly(TETA/CBA) carriers.

\subsection{3. miRNA}

siRNA and miRNA share some similarities in that both oligonucleotides possess duplex forms and need to form RISC for gene regulation. However, whereas the siRNA is highly specific to one target gene, the miRNA is capable of recognizing multiple mRNA targets. ${ }^{87}$ Previous studies reported that more than $60 \%$ of all protein-coding genes have at least one conserved binding site with miRNAs. ${ }^{88}$ Moreover, approximately $50 \%$ of miRNA-targeting genes are found at cancer-associated genomic regions or fragile sites. ${ }^{89}$ By taking advantage of the miRNA's multi-targeting capability and close relationship with cancer diseases, miRNA therapy can become a promising treatment for multigenic diseases, including various types of cancers. ${ }^{90}$

By delivering miRNAs complexed with cationic polymers, it 
was demonstrated that multiple genes associated with colon carcinoma can be regulated simultaneously. ${ }^{91}$ If only one gene is regulated to treat multigenic diseases, it may have a less therapeutic effect. As cancer is involved with highly complicated mechanisms, delivery of multi-targeting miRNAs would behighly effective. In this study, chemically unmodified miRNAs were complexed with cationic PEIs, which displayed the improved therapeutic effects in a mouse model of colon carcinoma. The therapeutic effect of two different miRNAs, miR-145 and miR-33a, were investigated; miR-145 has an important role in reducing tumor proliferation and increasing apoptosis, and miR-33a is capable of downregulating the oncogenic kinase, Pim-1. After PEI/miR145 complexes were injected into a xenograft mouse model, almost $50 \%$ decrease of tumor volume was detected over 23 days. In contrast, the injection of PEI/nonspecific RNA complexes resulted in an approximately 15-fold increase in tumor volume. The PEI/miR-33a treatment was also effective to reduce tumor growth. When the PEI/miR-33a was treated to a tumorbearing mouse, approximately $40 \%$ reduction of tumor growth was observed in comparison with the PEI/nonspecific RNA as a negative control.

\subsection{4. mRNA}

Compared to other oligonucleotides for gene silencing, mRNAs are delivered into the cells for protein expression, holding broader therapeutic potential. However, as the mRNAs are significantly large and hydrophilic, their intracellular delivery is generally more challenging than that of other therapeutic oligonucleotides. The mRNAs contain open reading frames (ORFs), along with 5' capping and 3' poly-A tailing, to be translated by ribosome. Their molecular weight roughly ranges from 300 to $5000 \mathrm{kDa}$, which is much larger than that of other oligonucleotides in therapeutics (ASO: 4 10 kDa and siRNA: 15 kDa). ${ }^{92}$ Some early studies have demonstrated the ability of naked mRNAs when internalized into cells via scavenger-receptor mediated endocytosis. ${ }^{93}$ However, it is challenging for the naked mRNAs to diffuse across cell membranes, due to their large size and negatively charged backbones. Nevertheless, the advantage of mRNA delivery is clear; compared to pDNAs, mRNAs are used for transient, yet rapid expression of therapeutically useful proteins, making the mRNAs highly valuable for a safe and fast gene therapy. ${ }^{94}$ Currently, several studies of mRNA delivery have focused on therapeutic applications, such as vaccination, protein therapy, and gene editing. ${ }^{95,96}$

To load the large mRNAs, polymeric carriers, which are biocompatible and biodegradable, are inherently necessary to be composed of large-sized polymers. The terpolymer of $\operatorname{poly}(\beta$ amino ester)-co-poly- $\varepsilon$-caprolactone (pBAE-co-PCL) was developed as an mRNA carrier through ring open polymerization (ROP) ${ }^{97}$ The pBAE is suitable for mRNA delivery; this cationic polymer is less toxic than others to cells and can be synthesized from more than 2000 libraries with no byproducts, allowing easy post-modifications. ${ }^{98}$ The PCL is a commonly used biodegradable and biocompatible polymer for oligonucleotide delivery, due to its high colloidal stability and facile cellular uptake by endocytosis. ${ }^{17}$ The pBAE-co-PCL-based terpolymer was complexed with the mRNAs coding for firefly luciferase, and the result- ing complex was given to mice by intravenous (IV) injection. As a result, it showed a 5-times higher transfection efficiency in spleen than the mRNAs complexed with jetPEI, a commercial transfection reagent.

Recently, there have been several studies to treat lung-related diseases using a drug delivery system (DDS) through inhalation. ${ }^{99}$ Unlike the DDS by IV injection, that by inhalation accompanies a nebulization process, making the inhalation delivery challenging. Polymeric nanoparticles are more suitable for the inhaled delivery than other nanoparticles, such as lipid-based nanoparticles, due to their higher resistance to the shear force caused by the nebulization. ${ }^{100}$ For the inhaled mRNA delivery, hyperbranched pBAE has been developed and applied to the treatment of lung-related diseases. ${ }^{101}$ The branched structures of pBAE are more stable and have greater positive surface charge than its linear structures, so the use of the hyperbranched structures could be applicable for inhalation. When the hyperbranched pBAE with the mRNAs coding luciferase genes were inhaled every three days, 10-times larger luciferase proteins were produced compared to the linear PEI, and no symptom of local and systemic toxicity was observed.

To maximize mRNA transfection efficiency, one study controlled the end-group composition and molecular weight of poly(amine-co-ester) (PACE), which is composed of cationic diol monomers, lactone monomers, and diacid monomers. ${ }^{102}$ Cationic diol monomers provide a low cationic charge density, leading to electrostatic interactions with mRNAs when lactone monomers stabilize the polyplex using hydrophobic interactions. In addition, use of diacid monomers allows the main chain of the polymer to contain biodegradable ester bonds. Previously, the same group demonstrated the therapeutic efficiency of PACE/pDNA polyplexes, ${ }^{103}$ and at this time, the dependence of mRNA transfection efficiency on the molecular weight and end-group composition of PACE was thoroughly clarified. As the molecular weight of PACE decreased from $20 \mathrm{kDa}$ to $5 \mathrm{kDa}$, the transfection efficiency of mRNAs increased 100-fold. This tendency reached a plateau at $5 \mathrm{kDa}$, but the transfection efficiency at $2 \mathrm{kDa}$ dramatically dropped, providing the guideline in choosing the molecular weight of PACE for efficient mRNA delivery. In addition, methylamino ethanol (-MAE) and carboxyl ($\mathrm{COOH}$ ) groups as end-groups of PACE were investigated in the same work. The result showed that $5 \mathrm{kDa}$ PACE-MAE encapsulated $98 \%$ of mRNAs, while PACE-COOH of the same MW did only $18 \%$, indicating the end-group dependency in mRNA loading.

\subsection{Stimuli-responsive polymeric carriers}

Even after low in vivo stability and short circulation time of therapeutic oligonucleotides are addressed well by use of polymeric carriers, the carriers can be further evolved to be stimuli-responsive; the stimuli-responsive carriers can be highly useful for preventing side effects and improving transfection efficiency, thereby enhancing therapeutic effects. ${ }^{104}$ If specifically loaded molecules are selectively released at a desired location and time, the smart carriers can avoid potential side effects to healthy tissues and organs. Moreover, their capability to selectively unload the delivered oligonucleotides within endosome is valuable for 
improving the transfection efficiency of the oligonucleotides. For therapeutic applications, the stimuli that polymeric carriers can be responsive to could be divided into two categories: (1) endogenous stimuli, such as $\mathrm{pH}$, redox levels, and enzyme concentrations, and (2) exogenous stimuli, such as heat, ultrasound, light, and magnetic field. The endogenous stimulus response system can achieve precise spatial control by spontaneously releasing the loaded molecules when the oligonucleotide-carrying carrier arrives at the disease-specific environments. On the other hand, the exogenous stimulus response system requires external triggers, but it can exhibit a significantly high transfection efficiency and temporal control accuracy through immediate release at the local area with the external stimuli.

\subsubsection{Endogenous stimuli}

The environment of abnormal cells, such as tumors, differs from that of normal cells; for example, most tumor cells exhibit relatively low $\mathrm{pH}$, produce abundant $\mathrm{ROS}$, and overexpress specific enzymes, compared to normal cells. ${ }^{35}$ When polymeric carriers are designed to respond to the different environment in releasing therapeutic oligonucleotides, the therapy can be localized to the targeted cells, leading to higher efficacy and efficiency in therapeutic effects. Considering the therapeutic mechanisms of various oligonucleotides (Figure 2), the endosomal escape of the delivered oligonucleotides is significantly important, and, there have been the advancement of polymeric carriers in response to the distinct environment within the endosome. ${ }^{105}$

\subsubsection{1. pH}

pH can be a crucially important stimulus in biological systems as it is an intrinsic property of various diseases, pathological conditions, and cellular microenvironments. ${ }^{106,107}$ In particular, by endocytosis, a cellular uptake process, drug carriers are typically internalized into the endosome of cells, and the endosome undergoes a maturation process to lysosome, progressively making the internal $\mathrm{pH}$ decrease up to $\mathrm{pH} 4.5 .^{108}$ Considering that the $\mathrm{pH}$ of the cytoplasm is 7.4, the endosome is a unique environment to serve as a trigger of $\mathrm{pH}$-responsive system that selectively releases delivered oligonucleotides at low $\mathrm{pH}$. Moreover, compared to physiological $\mathrm{pH}(\sim 7.4)$, the $\mathrm{pH}$ around tumor and inflammatory cells is lower $(6.6 \sim 6.8),{ }^{52}$ so the polymeric carriers capable of responding to low $\mathrm{pH}$ enable spatial control in the release of therapeutic oligonucleotides. Given the intrinsic $\mathrm{pH}$ of the physiological and pathological microenvironments, the oligonucleotide delivery based on the polymeric carriers with $\mathrm{pH}$-responsive moieties can be an excellent way to improve the therapeutic efficiency of the delivered oligonucleotides without adverse effects.

When polymeric carriers include pH-responsive linkers, they can enhance cellular uptake by breaking down the size of the carriers near desired locations, such as tumor cells. Even though PEGylation improves the in vivo stability of therapeutic oligonucleotides, it lowers the cellular uptake of the resulting large particles. ${ }^{109}$ The siRNA-loaded polymeric nanoparticles wherein PEG is linked by an acid-cleavable amide bond (Dlink) were developed, and their behavior near tumor cells was subsequently investigated. ${ }^{110}$ In response to acidic $\mathrm{pH}$, the Dlink- containing nanoparticles increased the release rate of siRNAs by $60 \%$ compared to the nanoparticles permanently linked with PEG. The pH-responsive displacement of PEGs from the Dlink-containing nanoparticles was effective to improve their gene silencing efficiency; while the silencing efficiency of Plk1 siRNAs was $66.9 \%$ at $\mathrm{pH} 7.4$, it increased to $80.2 \%$ at $\mathrm{pH} 6.5$. Moreover, when the same dose was administered to mice, the tumor weight decreased from $0.54 \mathrm{~g}$ to $0.29 \mathrm{~g}$, confirming that the acid-cleavable linker system can exhibit high tumor suppression efficacy according to its $\mathrm{pH}$-responsive ability.

Chemically modified cationic polymers can be used to promote oligonucleotide releasing by inducing disruption of endosomal membrane at low $\mathrm{pH}^{111}$ Positively charged PEI has been widely used due to its high loading capacity of oligonucleotides, and its proton sponge effect is attracting the strong attention for $\mathrm{pH}$-responsive oligonucleotide releasing. ${ }^{42}$ This effect has been observed in certain cationic polymers containing protonatable groups by endosomal $\mathrm{pH}$. When endosomal acidification proceeds, the polymeric carriers are further protonated, and counter ions (e.g., $\left.\mathrm{Cl}^{-}\right)$and water enter the endosome to neutralize them. For this reason, the endosome becomes swollen and eventually ruptured, making the internalized oligonucleotides diffuse in cytoplasm. To exploit the protonation by endosomal acidification, a PEI derivative, poly[N-[N-(2-aminoethyl)-2aminoethyl]aspartamide] (p[Asp(DET)]) has been developed, and its transfection efficiency of delivered oligonucleotides was examined (Figure 4(A)). ${ }^{112}$ This polymer exhibited two different $\mathrm{pKa}$ values, resulting in diprotonation at endosomal $\mathrm{pH}$, so it strongly interacted with vesicular membranes, thereby efficiently releasing the carried oligonucleotides through membrane disruption. In an in vivo test to mice, this polymeric carrier achieved nearly $600 \%$ decrease in target protein expression by successful oligonucleotide release into cytoplasm. In addition to the $\mathrm{p}[\mathrm{Asp}(\mathrm{DET})]$, various cationic polymers, including poly $(N, N$ dimethylamino ethyl methacrylate) (pDMAEMA), have been evaluated for their in vitro and in vivo uses. ${ }^{113}$

\subsubsection{Redox level}

There are many different redox agents, such as nicotinamide adenine dinucleotide phosphate, and GSH in the extracellular or intracellular environments of disease-related tissues and cells, and these redox agents play various roles including modulation of cell growth, differentiation, and even death. ${ }^{114}$ Among them, the most well-known reducing agent would be GSH, of which level is twice higher in the intracellular environment (i.e., endosome) than in the extracellular environment, and importantly, the GSH level increases up to 1000 times in disease-related milieu. ${ }^{115}$ As ROS involves in cellular signaling, pathogenic resistance, homeostasis, immunity, cell growth and differentiation, it has been also therapeutically important; it is known to be overexpressed near several disease cells. ${ }^{116}$ Therefore, there have been many studies to enable spatially controllable release of oligonucleotides by responding to the disease-specific redox level change. ${ }^{117,118}$

By incorporating disulfide bonds into polymers, the oligonucleotide carriers can be responsive to intracellular milieu, which can be a great way to enhance the blood circulation time and the cellular uptake of therapeutic oligonucleotides together. ${ }^{119,120}$ 
While high molecular-weight polymers can be advantageous for avoiding renal filtration, low molecular-weight polymers can be more easily internalized into cells. This means that to improve the therapeutic efficacy, the polymeric carriers should not be degraded outside cells, but selectively inside the cells. This selective degradation capability can be achieved by the development of disulfide bond-containing polymers, which can be separated into low molecular-weight polymers by GSH-induced reduction at specific locations. ${ }^{121,122}$ To assess the therapeutic usefulness of the spatioselective cleavage, three different types of polymeric carriers were synthesized; while two PEI polymers had different molecular weight (PEI25: $25 \mathrm{kDa}$ and PEI1.8: 1.8 $\mathrm{kDa}$ ), the third one (sSPEI1.8) was prepared by linearly linking $1.8 \mathrm{kDa}$ PEI through cleavable disulfide linkers, thereby yielding a molecular weight of $25 \mathrm{kDa}^{119}$ Compared to the cytotoxic PEI25, PEI1.8 showed the improved cell viability (up to 250\%), and interestingly, the effect of SSPEI1.8 was similar with that of PEI1.8. Despite the similar size, ssPEI1.8 carrying oligonucleotide drugs exhibited 3 times higher transfection efficiency than PEI25. Similar trends have been observed in various in vitro and in vivo assessments. ${ }^{123-125}$

The level of ROS, such as superoxides, hydrogen peroxides, peroxyl radicals, and hydroxyl radicals, can be also great to trig- ger the spatially controlled oligonucleotide release; the ROS involves in regulating cell signaling, but abundant ROS can induce oxidative stress, by which various diseases (e.g., cancer, inflammation, cardiovascular disease, and Alzheimer's disease) can occur. ${ }^{126,127}$ These diseases have their own pathways to locally produce a high level of ROS, so the disease sites have 10 100 times higher ROS concentrations than normal tissues. ${ }^{128}$ An ROSresponsive polymeric carrier using poly(amine thioketal) (PATK) was used for targeted oligonucleotide delivery to cancer cells. ${ }^{129}$ The half-lives of the polymer were 48, 20, and 11 hours in $50 \mathrm{mM}$, $100 \mathrm{mM}$, and $200 \mathrm{mM} \mathrm{H}_{2} \mathrm{O}_{2}$ solutions, respectively, indicating that the degradation of PATK heavily depends on the $\mathrm{H}_{2} \mathrm{O}_{2}$ concentrations. Compared to non-cancerous cells, The PATK with oligonucleotide drugs displayed $\sim 60 \%$ higher transfection efficiency to cancerous cells, proving the potential of ROS-responsive polymers for disease-selective oligonucleotide delivery.

The exceptional stimulus specificity of ROS-responsive polymers enabled their application in oral oligonucleotide delivery (Figure 4(B)). ${ }^{130}$ In this work, siRNA-loaded thioketal nanoparticles were orally delivered to inhibit TNF- $\alpha$ on intestinal inflammation. With a daily oral gavage of the nanoparticles, biodistribution of siRNAs in the organs of mice was investigated. As a result, the orally delivered siRNAs were highly localized in the inflamed
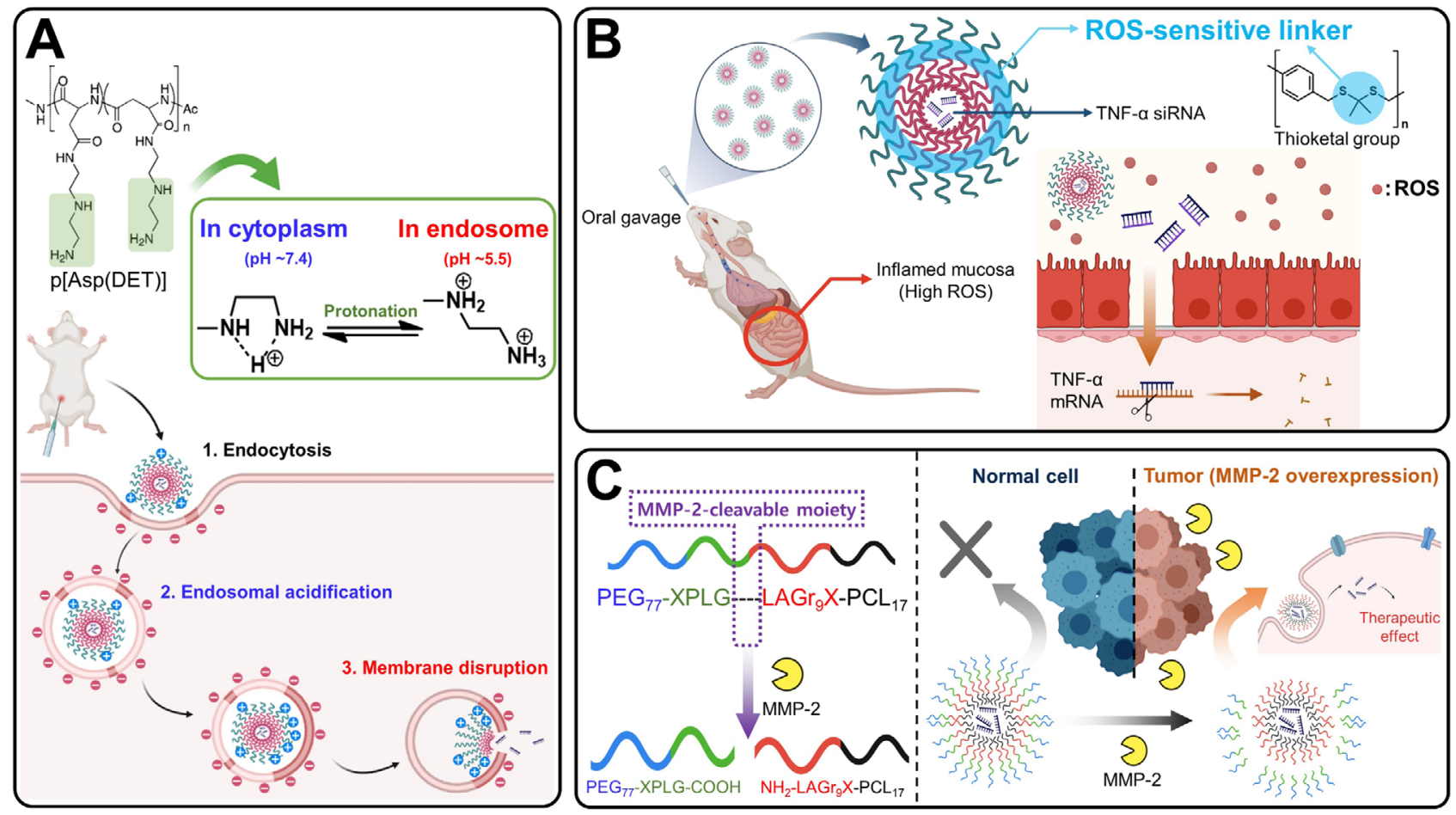

Figure 4. Development of stimuli-responsive polymers to improve the therapeutic efficacy of delivered oligonucleotides. (A) pH-responsive polymeric carrier using poly[N-[N-(2-aminoethyl)-2-aminoethyl]aspartamide] (p[Asp(DET)]). The p[Asp(DET)] has two different protonatable moieties; one is protonated at $\mathrm{pH} 7.4$, i.e., the $\mathrm{pH}$ of extracellular matrix and cytoplasm, and the other is protonated at $\mathrm{pH} 5.5$, which can be reached during endosomal acidification. The diprotonated polymer can induce membrane disruption by strongly interacted with vesicular membranes, thereby making therapeutic oligonucleotides escaped from the endosome. (B) ROS-responsive polymeric carrier system including thioketal groups. The thioketal group-including polymeric carriers respond to a high level of ROS on inflammation sites. Due to the specificity of the ROS-sensitive linkers, the ROS-responsive polymers enable oral oligonucleotide delivery. When the polymeric nanoparticles loaded with TNF- $\alpha$ siRNAs were administered to mice by oral gavage, the TNF- $\alpha$ siRNAs were exceptionally localized in the inflamed intestinal tissues, leading to effective degradation of TNF- $\alpha$ mRNAs. (C) Enzyme-responsive polymeric carrier system that contains cleavable moieties by matrix metalloproteinase 2 (MMP-2), one of the proteases. As the MMP-2 is generally overexpressed in disease cells such as cancer cells and inflammatory cells, the polymeric carriers can detach the polyethylene glycol (PEG) blocks in the presence of MMP-2 on tumors, promoting the cellular uptake of the smaller and more hydrophobic polyplexes. 
intestinal tissues; compared to the liver, the intestine had 3 times more populated siRNAs, confirming the selective release of the delivered siRNAs in the inflamed tissues. The transfection efficiency of the thioketal nanoparticles was significantly high; as the transfected TNA- $\alpha$ siRNA was used to degrade the TNF- $\alpha$ mRNA, the amounts of TNF- $\alpha$ mRNAs was decreased by 10 times compared to the siRNA delivery without the ROSresponsive carriers. As the high level of ROS is a major and unique marker of diseases, a variety of ROS-responsive, polymeric oligonucleotide carriers have been studied as safe and efficient delivery systems. ${ }^{129,131,132}$

\subsubsection{Enzyme concentration}

As biocatalyst, enzymes involve in various reactions in our bodies. The biochemical processes are performed at specific conditions due to the environment-dependent activity of the enzymes, and to initiate the reactions, the biological enzymes are necessary to recognize target substrates selectively. ${ }^{133,134}$ Moreover, dysfunctions of cells can cause some enzymes to be highly overexpressed in tumors and inflammatory tissues. Such disease-relevant enzyme overexpression is significantly useful for developing stimuli-responsive systems, including smart oligonucleotide drug delivery. For example, when polymeric carriers are synthesized to include substrate-mimicking moieties, they can be recognized by the enzymes overexpressed by disease cells. The enzyme-responsive polymeric carriers would be valuable for lowering the side effects, but increasing the therapeutic efficiency through releasing oligonucleotide in targeted sites only. ${ }^{135,136}$

Proteases that involve in cell proliferation, invasion, and apoptosis are one of the most suitable enzymes for enzymeresponsive oligonucleotide delivery as they are upregulated in diseases such as cancer and inflammation. ${ }^{137}$ Among various proteases, matrix metalloproteinase 2 (MMP-2) was chosen to develop siRNA-loaded polymeric carriers; ${ }^{138}$ the MMP-2 is an enzyme engaged in the degradation of extracellular matrix as overexpressed by almost all tumors. ${ }^{35,139,140}$ To make the polymeric carrier recognizable as the substrate of MMP-2, MMP-2degradable peptides, LAGs, were used as bridges between PEGs and PCLs within the synthetic polymer (Figure 4(C)). In the presence of MMP-2, the LAG bridges were successfully cleaved, so PEG chains were subsequently displaced from the polymeric carrier. This PEG displacement resulted in reducing the size of siRNA-loaded carrier, thereby improving its cellular uptake. In in vivo mice model, the MMP-2-responsive polymeric carrier selectively delivered siRNAs into tumor tissues, reducing the tumor volume by $63 \%$. Due to the environment specificity, various enzyme-responsive systems have been recently developed for effective oligonucleotide delivery. ${ }^{141}$

\subsubsection{Exogenous stimuli}

By artificially generated external stimuli, spatiotemporal control of oligonucleotide-releasing system is achievable to increase the therapeutic efficiency of oligonucleotides. For clinical applications, a large dose of oligonucleotides is administered to patients for a long time to result in a practical therapeutic effect. Moreover, in terms of inter-patient variability, the levels of endoge- nous stimuli can be quite different among individuals, making the same endogenous stimuli-responsive system not generally applicable for all the patients. ${ }^{142}$ To solve these problems, exogenous stimuli can be exploited in developing the smart oligonucleotide drug carriers; it is possible to precisely control the release of oligonucleotides by adjusting various parameters, including exposure time of heat, frequency of ultrasound, and wavelength of light. ${ }^{104,114,118}$ Moreover, when the oligonucleotide drug carriers are intravenously administered, they can release a high concentration of the therapeutic oligonucleotides at the local area where various external stimuli are applied during blood circulation.

\subsubsection{Heat}

In pathological environments, such as tumors, the temperature can be slightly higher than that in normal physiological environments; ${ }^{143}$ for example, malignant tumors show the temperature of $40 \sim 42{ }^{\circ} \mathrm{C}$, while the temperature around normal tissues is $36.5 \sim 37.5{ }^{\circ} \mathrm{C}$ for human beings. However, this temperature difference may not be large enough for oligonucleotide drug carriers to use as a disease-relevant stimulus, so raising the local temperature by external heating has been extensively considered instead. ${ }^{144,145}$ Importantly, increasing the local temperature cannot only induce a direct cytotoxic effect in tumors, but also enhances the permeability of the tumors, thereby facilitating oligonucleotide penetration. ${ }^{146,147}$ Some polymers undergo a phase transition, changing hydrophilicity and hydrophobicity at the lower critical solution temperature (LCST), ${ }^{16}$ and their thermos-responsive behaviors are appropriate for selective oligonucleotide release. ${ }^{148,149}$ Above the LCST, dehydration occurs as the hydrophobicity of the polymeric carriers increases, and the tight complexes are formed between oligonucleotides and the polymers. These complexes can prevent degradation of the loaded oligonucleotides and simultaneously improve the cellular uptake due to their compact structures. In contrast, under the LCST, hydrogen bonds are newly formed between the polar compartments of the polymers and water molecules, so the carried oligonucleotides can be subsequently dissociated from the polymeric carriers, which is highly advantageous for the following transcription and translation. ${ }^{150}$

Poly(N-isopropylacrylamide) (PNIPAM) is a representative LCST-type temperature-responsive polymer, widely known for its LCST at $32{ }^{\circ} \mathrm{C} .{ }^{151}$ However, by adjusting the ratio of hydrophobic and hydrophilic groups, the LCST is controllable for desired therapeutic applications. ${ }^{152}$ A copolymer of PEI-PNIPAM was synthesized for thermo-responsive oligonucleotide delivery (Figure 5(A)), and to increase the transition temperature up to $42{ }^{\circ} \mathrm{C}$, vinylpyrrolidone was introduced in the copolymer as a hydrophilic monomer. ${ }^{153}$ When heat was applied, the polymeric carrier with luciferase genes increased the gene expression level by more than 2 orders of magnitude, compared to no heating. In an in vivo test of mice, the polymeric carriers with pDNAs were injected into the tumor-bearing legs, which were incubated in a hot water bath at $42{ }^{\circ} \mathrm{C} .{ }^{154}$ As a result, the transfection efficiency was more than 10 times higher than the negative control, the mice without water bath incubation. In a similar way, temperature-responsive polymers consisting of synthetic polymers and polypeptides have been exploited to 
increase the transfection efficiency of delivered oligonucleotides through heating treatment. ${ }^{155}$

\subsubsection{Ultrasound}

Using ultrasound, spatial and temporal control of oligonucleotide delivery system is also attainable, preventing unnecessary side effects on healthy tissues. This non-invasive stimulus is quite attractive due to the easy control of frequency, exposure time and duty cycles, achieving personalized ultrasound intensities. ${ }^{156}$ Ultrasound-responsive oligonucleotide carriers were constructed by the combination between cationic polymers and microbubble-filled liposomes (Figure 5(B)); ${ }^{157}$ when ultrasound is applied to the carriers, the encapsulated microbubbles rapidly expand, compress, and collapse, and this vigorous movement can cause cavitation and sonoporation, mediating the oligonucleotide carrier destabilization and cell membrane permeabilization. ${ }^{156,157}$ Using these phenomena, the delivered therapeutic oligonucleotides can be readily liberated from the carriers and enter the cell through the transient pores formed by the cavitation and sonoporation, thereby enhancing the transfection efficiency. In a study, to maximize the transfection efficiency of DNAs, various parameters such as acoustic pressures, microbubble concentrations, and DNA dosages have been tested. ${ }^{158}$ At an optimal condition, the ultrasound treatment increased the gene expression level by 100 times compared to no treatment. Along with the ultrasound, various stimuli, such as $\mathrm{pH}$ and temperature, have been exploited together to develop dual or multi-responsive oligonucleotide carriers. ${ }^{159}$

\subsubsection{Light}

Light is also an exogenous and non-invasive stimulus applicable for the oligonucleotide delivery by adjusting its wavelength, polarity, intensity and duration. Currently, ultraviolet (UV) has been frequently used as the external stimulus for selective release of therapeutic oligonucleotides, even though the penetration depth of UV radiation in human skin is quite shallow. ${ }^{160,161}$ The UV has more energy than the other types of light, so it can easily induce chemical cleavage and isomerization of various UV-
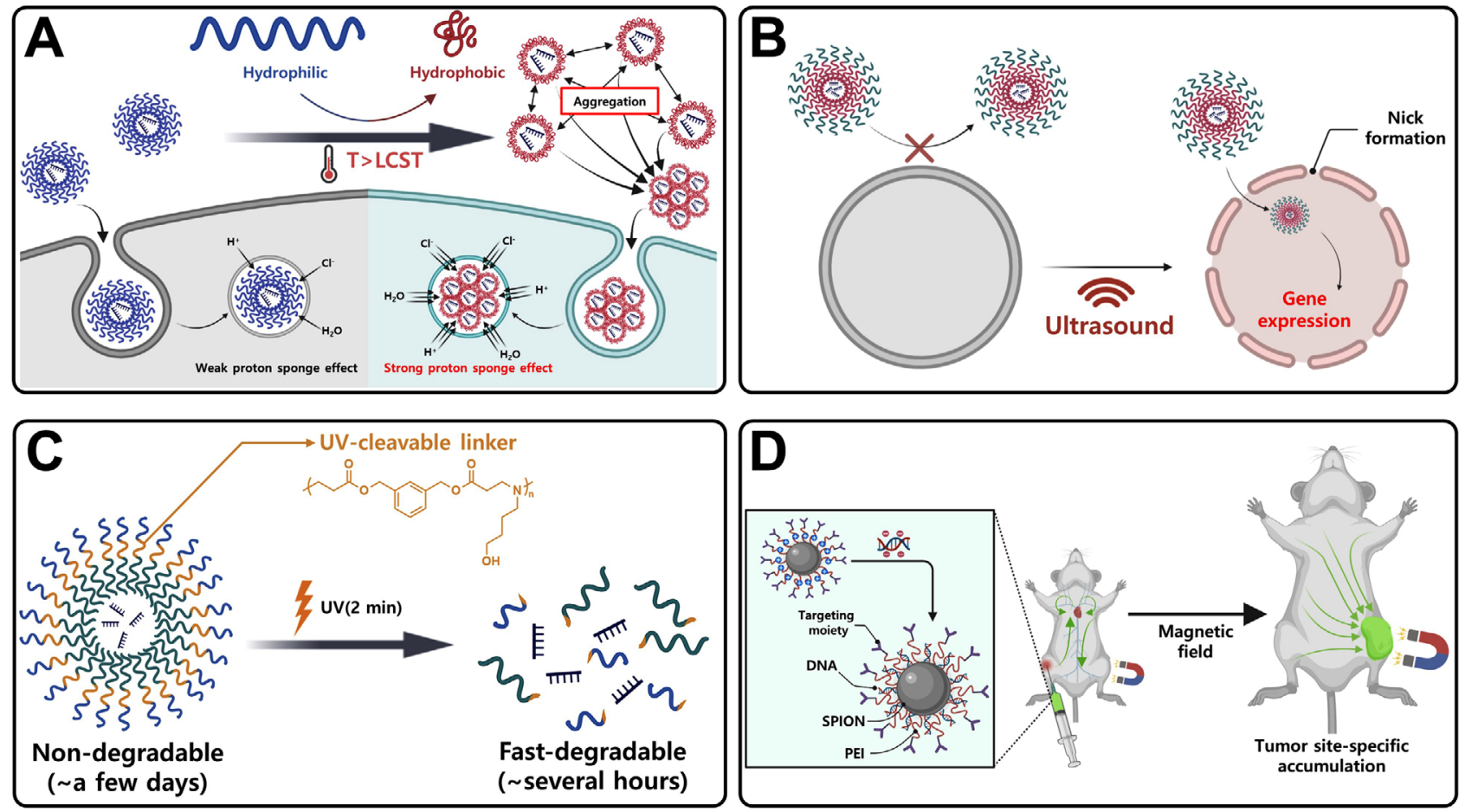

Figure 5. Development of polymeric carriers that utilize exogenous stimuli for selective release of oligonucleotides. (A) Heat-responsive polymeric carriers using poly(N-isopropylacrylamide) (PNIPAM)-PEI to enhance cellular uptake and endosomal escape of oligonucleotides. PNIPAM is one of the representative temperature-sensitive polymers featuring lower critical solution temperature (LCST). When the temperature becomes higher than LCST by external heating, the PNIPAM undergoes hydrophilic-hydrophobic phase transition, resulting in globulization of polymer chains in an aqueous solution. Due to hydrophobic interactions, the polymeric carriers can be aggregated each other, followed by the endocytosis of the resulting aggregates. Compared to individual polymeric carriers (left), the aggregated ones (right) are eager to make a stronger proton sponge effect; in this way, by heat stimuli, the heat-responsive polymers enhance the endosomal escape of the delivered oligonucleotides. (B) Ultrasound-responsive polymeric carriers constructed by the combination between cationic polymers and microbubble-filled liposomes. With the ultrasound, the microbubbles in the polymeric carriers can cause to form small pores at cell membranes through sonoporation and cavitation. Through the pores, the delivered oligonucleotides easily enter the cells, thereby improving transfection and therapeutic efficiency. (C) UV-responsive polymeric carriers based on poly( $\beta$-amino ester) (pBAE). When non-degradable pBAE chains are synthetized to include UV-cleavable nitrobenzyl groups, the resulting polymeric carriers can be rapidly decomposed upon external UV irradiation, which can be synchronized with the release of encapsulated oligonucleotides. (D) Magnetic field-responsive nano-composites for therapeutic oligonucleotide delivery. When the surface of superparamagnetic iron oxide nanoparticles (SPIONs) is modified with PEI, therapeutic oligonucleotides, such as miRNA-encoding plasmid DNAs (pDNAs) can be well-loaded on the magnetic nano-composites. When the SPIONs with pDNAs are injected into a body, they can be selectively localized on tumor sites during blood circulation by use of a magnet. Due to the high concentrations of pDNAs around the tumors, their transfection efficiency can be improved with the increase of miRNA transcription. 
reactive moieties, which is highly suitable for selective degradation of polymeric carriers and oligonucleotide release. ${ }^{104,162}$ For instance, isomeric transformation of azobenzene group by UV can be used as a trigger for the oligonucleotide release of polymeric carriers to increase the efficiency of oligonucleotide transfection. ${ }^{163,164}$ When irradiated with $350 \mathrm{~nm}$ UV light, the azobenzene group is converted from a hydrophobic trans-isomer to a hydrophilic cis-isomer, and in the presence of visible light at $436 \mathrm{~nm}$, it is converted back to the trans form. The hydrophobic transisomers can facilitate dense and stable packing of oligonucleotides within the polymeric carrier, but upon UV exposure, a large number of oligonucleotides can be rapidly released as the formation of hydrophilic cis-isomers causes unpacking of the hydrophilic oligonucleotides. For a light-responsive oligonucleotide delivery carrier, the polymer composed of a central azobenzene moiety and two terminal pDMAEMA blocks (Azo-pDMAEMA) was synthesized, and its transfection efficiency was examined by an in vitro cell test. ${ }^{165}$ With UV irradiation on the Azo-pDMAEMA, the delivered oligonucleotides were transfected into the cells more efficiently than without irradiation, yielding up to 2.7 times higher efficiency. The cytotoxic effect of the UV irradiation was minimal; there was no significant change in the cell viability when measured after 15-minute UV exposure.

A UV-cleavable nitrobenzyl group can be introduced into a polymeric carrier to accelerate its degradation for the immediate release of therapeutic oligonucleotides. pBAE is widely used for the material of oligonucleotide delivery carriers due to its synthesis easiness and versatility. However, this ester linkage-containing polymer is too slowly degraded by hydrolysis; the degradation takes several hours to a few days under cellular conditions, which would not be appropriate for the therapeutic application with high concentrations of oligonucleotides. ${ }^{166}$ The 2-nitrobenzene moiety was introduced into the backbone of pBAE for on-demand oligonucleotide release (Figure $5(\mathrm{C}))^{167}$ This oligonucleotide carrier improved transfection efficiency about 70\% upon 2-minute UV irradiation and showed negligible cytotoxicity. Similarly, other types of light-responsive linkers have been introduced to various polymers, which would be highly useful to rapidly release a high concentration of oligonucleotides without notable toxicity. ${ }^{168,169}$

\subsubsection{Magnetic field}

When synthetic polymers are combined with magnetic nanoparticles, the resulting nano-composites can be the great carriers capable of localizing oligonucleotides in a body as guided by a magnetic field, another non-invasive stimulus. For example, by applying the exogenous magnetic field to tumors, the magnetic nanoparticles during blood circulation can be concentrated near the disease sites. However, as unmodified nanoparticles cannot readily capture therapeutic oligonucleotides, surface modification with cationic polymers, such as PEI, N-acylated chitosan, and polylysine, should be performed to electrostatically load the negatively charged oligonucleotides. ${ }^{170}$ Superparamagnetic iron oxide nanoparticles (SPIONs) have been chemically coated with chondroitin sulfate-PEI copolymers for miRNA-encoding pDNA delivery (Figure 5(D)). ${ }^{171}$ While the PEIs hold the pDNAs for miRNA-128 transcription on the surface of the SPIONs, the chondroitin sulfates were labeled to the nano-composites for active tumor targeting and CD44-mediated endocytosis. In the presence of the magnetic field, the site-specific accumulation of the nano-composites were successfully induced; when a static magnet was placed on the xenografted tumors of mice after injection of nanocomposites, the localized biodistribution of the injected nanocomposites was observed within 3 hours, along with the improved transfection of delivered pDNAs. As the magnetic nanoparticles allow further in vivo imaging, the oligonucleotide-carrying nano-composites can be also useful for theranostic applications. ${ }^{170,172}$

\section{In vivo targeting by combination of oligonucle- otides and polymers}

Even though synthetic polymers can be prepared to be highly responsive to many different types of endogenous and exogenous stimuli, one of the most deficient abilities as effective oligonucleotide carriers would be disease site targeting. ${ }^{173}$ To maximize therapeutic efficacy with minimal adverse effects, the therapeutic oligonucleotides should be delivered to the only targeted cells, which are encircled by their own specific surface receptors. ${ }^{174}$ To empower the polymeric carrier to specifically recognize target cells, many different types of ligands, such as monoclonal antibodies, ${ }^{175}$ receptor-specific peptides, ${ }^{176}$ and nucleic acid aptamers, ${ }^{57}$ have been employed. Among these ligands, aptamers are the chemically synthesized oligonucleotides, and as they fold into desired conformations at specific conditions, they are capable of recognizing many kinds of targets including cell surface receptors. ${ }^{56}$ Interestingly, the denaturation of their 3D conformations is reversible, so unlike irreversibly denatured, protein-based ligands, further chemical modifications in harsh conditions are allowed, making the aptamers readily conjugated with a variety of highly structured polymers. ${ }^{177}$ Due to the conjugation availability between the aptamers and the polymers, tree-like or star-like dendrimers and hyperbranched polymers have been decorated with the synthetic aptamers, and by the multivalency effect of the polymer-decorating aptamers, the resulting hybrid carrier exhibited the enhanced targeting capability, revealing the potential for target-specific oligonucleotide delivery. ${ }^{178}$

By chemically conjugating cancer cell-targeting aptamers with the PEG-based polymers capable of controlled drug release, aptamer-polymer hybrid (APH) molecules $(<10 \mathrm{~nm}$ in diameter) were created for the programmed drug release only into cancer cells. ${ }^{179}$ Using click chemistry, one nucleolin-specific aptamer and one block copolymer, composed of ethylene glycol and ethylene glycol vinyl glycidyl ether, were site-specifically conjugated to produce the APH molecule; even after the conjugation reaction in organic solvents, the aptamer within the APH maintained the high affinity to target cancer cells. Moreover, multiple chemotherapy drugs, doxorubicin, were further conjugated to the polymer through enzyme-cleavable linkers with a high yield, so when the APH was internalized in the cell, the delivered drugs could be selectively released due to the linker cleavage by endosomal esterases. The APH specifically recognized breast cancer cells that overexpressed nucleolin on their cell 
surfaces, and successfully released the chemotherapy drugs within the endosomes of the only targeted cells, decreasing the cell viability up to $64 \%$. In contrast, the healthy cells were not targeted at all by the APH, and no internalization and drug release was observed, indicating the minimal side effect by the use of the APH drug carrier.

Rather than a single aptamer, multiple cancer-specific aptamers have been conjugated to a poly(amidoamine) (PAMAM) dendrimer nanocarrier to improve cell targeting and transfection efficiency. ${ }^{180}$ The PAMAM dendrimer possessed a large empty inner space, which could load hydrophobic drugs, such as camptothecin (CPT) that is commonly used for colorectal cancer treatment. At the sphere surface of the dendrimer, functional end groups were located, so a number of aptamers could be conjugated for surrounding the dendrimer carrier, leading to enhancement of binding interactions with target receptors. When the aptamer-decorated dendrimer loaded the CPT, the encapsulation efficiency was almost $100 \%$, and the improved target binding and cytotoxicity to colorectal cancer cells were observed in in vitro and in vivo experiments.

\section{Application of oligonucleotide-polymer polyplex to immunotherapy}

Currently, the treatment paradigm for cancer is rapidly changing from chemotherapy, the treatment by chemical substances, to immunotherapy in which immune cells are activated for killing malignant cells. ${ }^{181,182}$ In promoting the ability of T-cells to specifically target cancer cells, chimeric antigen receptor (CAR) genes are necessary to be transfected into the T-cells, and the simultaneous delivery of both antigens and $\mathrm{CpG}$ oligodeoxynucleotides to the immune cells would be effective to activate the immune system in our bodies. ${ }^{183,184}$ To address this need, there have been different types of polymeric carriers for co-drug delivery, which have great advantages in drug loading capacity compared to viral vectors that have a limit on the size of cargo drug (e.g., $\sim 10 \mathrm{~kb}$ for lentiviral vectors). ${ }^{185}$ Moreover, the polymeric carriers can be functionalized to be responsive to various stimuli, such as $\mathrm{pH}$; for instance, selective endosomal release of delivered oligonucleotides is achievable for increasing their transfection efficiency. In this last chapter, we introduced some examples of immunotherapy using copolymer oligonucleotide carriers.

Graft copolymers have been used to efficiently transfer CAR gene-encoding pDNAs or mRNAs into T-cells. ${ }^{186}$ T lymphocytes play a key role in recognition and clearance of antigens. However, tumors secrete several immunosuppressive cytokines, so T-cell activation is significantly interfered ${ }^{187}$ To avoid the interference of T-cell activation, the CAR gene is necessary to be transfected into the T-cells, and the genetically modified T-cells can specifically bind to the receptors of cancer cells, recovering the immune response. ${ }^{188}$ Comb-shaped and sunflower-shaped graft copolymers were developed to deliver pDNAs and mRNAs into T-cells and poly(2-hydroxyethyl methacrylate) and poly(2-(dimethylamino)ethyl methacrylate) were used to synthesized the graft copolymers with various molecular weights. The graft copolymers efficiently transfected pDNAs into T-cells (25-50\% in efficiency) compared to bPEI, of which transfection was negligible in serum-free medium. In particular, the comb-shaped graft copolymer showed successful transfection of primary human T-cells with higher efficiency than other types of copolymers, exhibiting $18 \%$ and $25 \%$ transfection by pDNAs and mRNAs, respectively.

By systematically designing the polymer to facilitate the endosomal escape of delivered antigens and adjuvants, the polymeric carrier dramatically improved the activation efficiency of immune systems. ${ }^{189}$ When the adjuvants, such as CpG oligonucleotides, are transferred along with antigens to antigenpresenting cells, the immune response is highly promoted ${ }^{184}$ In this work, the di-block copolymers with two multifunctional modules were synthesized to deliver ovalbumin (OVA) and CpG oligonucleotides. In a block, a small percentage of pyridyl disulfide ethyl methacrylate was conjugated with disulfide bonds to bind OVA antigens, and the positively charged DMAEMA containing tertiary amines was included to electrostatically interact with CpG oligodeoxynucleotides. In the other block, propylacrylic acids (PAAs) were introduced for $\mathrm{pH}$-responsive endosomal escape of delivered molecules. As the carboxylic acid residue was protonated at endosomal $\mathrm{pH}$, the PAA experienced a hydrophilic-to-hydrophobic transition, leading to membrane disruption. The di-block copolymers with both OVAs and CpGs showed 7 times higher T-cell response compared to the co-polymer only with OVAs. Moreover, the use of polymeric carriers was indeed effective, exhibiting 18 times higher response than the free use of OVAs and CpGs without carriers.

\section{Conclusion and perspectives}

To maximize the therapeutic effects of oligonucleotides and minimize their adverse effects simultaneously, the therapeutic oligonucleotides, such as ASOs, siRNAs, miRNAs, and mRNAs, have been systematically combined with a wide range of functionalized polymers. As the physical and chemical properties of the synthetic polymers were finely tuned, the resulting oligonucleotide carriers enabled loading of numerous oligonucleotides and their subsequent delivery to disease sites without renal clearance or nucleolytic degradation. Moreover, the polymeric carriers have been further engineered to respond to various endogenous and exogenous stimuli; the release of therapeutic oligonucleotides was highly localized to disease tissues, and the endosomal escape of the large oligonucleotides was significantly promoted, thereby increasing therapeutic efficacy and efficiency dramatically with reduced side effects. Currently, several clinical trials are ongoing based on the systematic combination of oligonucleotides and polymers, ${ }^{190}$ and the medical uses of the synergistic combination (e.g., immunotherapy) are also expanding. ${ }^{191}$

Even though oligonucleotides and polymers have complemented each other to actualize highly effective and efficient oligonucleotide therapy, several issues still remain. For example, inherent cytotoxicity of the polymer and relevant side effects should be mitigated. The cytotoxicity of polymeric carriers is typically attributed to their cationic surface charge and hydrophobicity, which are though essential for them to transfect disease cells with the therapeutic oligonucleotides. ${ }^{192}$ With a better 
understanding of cellular uptake and disease action mechanisms, various disease-specific stimuli could be further explored to develop the polymeric carriers that can become cationic or hydrophobic at desired locations only. The administered dose of the oligonucleotide-loaded polymers is also critical in terms of the side effects; by reducing the dosage, the side effects of oligonucleotides and polymers would be less influential, and importantly, the reduction of the medical care costs would be expected additionally. Moreover, as specifically folding oligonucleotides, i.e., aptamers, can recognize target cells like monoclonal antibodies, the incorporation of the synthetic affinity reagents can strengthen the power of synthetic polymers toward creation of target-specific oligonucleotide carriers. Furthermore, to solve the off-target effects of simply designed, therapeutic oligonucleotides, it can be considered to deliver the self-regulating oligonucleotides by polymeric carriers. By systematically incorporating toehold switches and riboswitches into the therapeutic oligonucleotides, the expression of the resulting oligonucleotides can be regulated by intracellular levels of disease-related biomolecules, ${ }^{30,193}$ which could be highly effective to increase the safety of oligonucleotide therapeutics in clinical use. With all efforts, we envision the development of safer and more effective combinations between therapeutic oligonucleotides and polymers, holding the potential for the next generation therapy even applicable for lots of rare or currently untreatable diseases.

\section{References}

(1) A. C. Walls, Y.-J. Park, M. A. Tortorici, A. Wall, A. T. McGuire, and D. Veesler, Cell, 181, 281 (2020).

(2) D. C. Wallace, Annu. Rev. Genet, 39, 359 (2005).

(3) T. A. Blauwkamp, S. Thair, M. J. Rosen, L. Blair, M. S. Lindner, I. D. Vilfan, T. Kawli, F. C. Christians, S. Venkatasubrahmanyam, and G. D. Wall, Nat. Microbiol., 4, 663 (2019).

(4) S. J. Chapman and A. V. S. Hill, Nat. Rev. Genet, 13, 175 (2012).

(5) M. L. Stephenson and P. C. Zamecnik, Proc. Natl. Acad. Sci., 75, 285 (1978).

(6) N. Nishiyama and K. Kataoka, Pharmacol. Ther., 112, 630 (2006).

(7) N. B. Tsui, E. K. Ng, and Y. D. Lo, Clin. Chem., 48, 1647 (2002).

(8) F. Iversen, C. Yang, F. Dagnæs-Hansen, D. H. Schaffert, J. Kjems, and S. Gao, Theranostics, 3, 201 (2013).

(9) J. Panyam and V. Labhasetwar, Adv. Drug Deliv. Rev., 55, 329 (2003).

(10) H. Cabral, K. Miyata, K. Osada, and K. Kataoka, Chem. Rev., 118, 6844 (2018).

(11) T. C. Roberts, R. Langer, and M. J. A. Wood, Nat. Rev. Drug Discov., 19 $673(2020)$

(12) D. C. Luther, R. Huang, T. Jeon, X. Zhang, Y.-W. Lee, H. Nagaraj, and V. M. Rotello, Adv. Drug Deliv. Rev., 156, 188 (2020).

(13) J. I. Cutler, E. Auyeung, and C. A. Mirkin, J. Am. Chem. Soc., 134, 1376 (2012).

(14) T. Thambi, Y. Li, and D. S. Lee, J. Control. Release, 267, 57 (2017).

(15) H. Park, K. Y. Lee, S. J. Lee, K. E. Park, and W. H. Park, Macromol. Res., 15, 238 (2007).

(16) S. Ashraf, H.-K. Park, H. Park, and S.-H. Lee, Macromol. Res., 24, 297 (2016).

(17) A. Kumari, S. K. Yadav, and S. C. Yadav, Colloids Surf. B, 75, 1 (2010).

(18) S. Alfei and S. Castellaro, Macromol. Res., 25, 1172 (2017).

(19) J. H. Kim, Y. T. Oh, K. S. Lee, J. M. Yun, B. T. Park, and K. T. Oh, Macromol. Res., 19, 453 (2011).
(20) C. Wang, S. Ravi, G. V. Martinez, V. Chinnasamy, P. Raulji, M. Howell, Y. Davis, J. Mallela, M. S. Seehra, and S. Mohapatra, J. Control. Release, 163, 82 (2012).

(21) H. J. Zhang, X. Zhao, L. J. Chen, C. X. Yang, and X. P. Yan, Talanta, 219, (2020).

(22) I. M. Verma and N. Somia, Nature, 389, 239 (1997).

(23) O. Boussif, F. Lezoualc'H, M. A. Zanta, M. D. Mergny, D. Scherman, B. Demeneix, and J. P. Behr, Proc. Natl. Acad. Sci., 92, 7297 (1995).

(24) R. W. Carthew and E. J. Sontheimer, Cell, 136, 642 (2009).

(25) A. Esquela-Kerscher and F. J. Slack, Nat. Rev. Cancer, 6, 259 (2006).

(26) A. B. Hill, M. Chen, C.-K. Chen, B. A. Pfeifer, and C. H. Jones, Trends Biotechnol, 34, 91 (2016).

(27) H. Yoo, H. Jo, and S. S. Oh, Mater. Adv., 1, 2663 (2020).

(28) J. Liu, Z. Cao, and Y. Lu, Chem. Rev., 109, 1948 (2009).

(29) A. D. Ellington and J. W. Szostak, Nature, 346, 818 (1990).

(30) S. V. Park, J.-S. Yang, H. Jo, B. Kang, S. S. Oh, and G. Y. Jung, Biotechnol. Adv., 37, 107452 (2019).

(31) I. Willner, B. Shlyahovsky, M. Zayats, and B. Willner, Chem. Soc. Rev., 37, 1153 (2008)

(32) T. Kim, K. Nam, Y. M. Kim, K. Yang, and Y. H. Roh, ACS Nano, 15, 1942 (2021).

(33) M. A. Mintzer and E. E. Simanek, Chem. Rev., 109, 259 (2009).

(34) F. Danhier, O. Feron, and V. Préat,J. Control. Release, 148, 135 (2010).

(35) K. Kessenbrock, V. Plaks, and Z. Werb, Cell, 141, 52 (2010).

(36) G. Park, B. Kang, S. V. Park, D. Lee, and Seung, Nucleic Acids Res., 49, 4919 (2021).

(37) R. L. Setten, J. J. Rossi, and S.-P. Han, Nat. Rev. Drug Discov., 18, 421 (2019).

(38) N. B. Y. Tsui, E. K. O. Ng, and Y. M. D. Lo, Clin. Chem., 48, 1647 (2002).

(39) K. Kawabata, Y. Takakura, and M. Hashida, Pharm. Res., 12, 825 (1995).

(40) I. Moret, J. Esteban Peris, V. M. Guillem, M. Benet, F. Revert, F. Dasí, A. Crespo, and S. F. Aliño, J. Control. Release, 76, 169 (2001).

(41) K. C. Cho, S. H. Choi, and T. G. Park, Macromol. Res., 14, 348 (2006).

(42) R. V. Benjaminsen, M. A. Mattebjerg, J. R. Henriksen, S. M. Moghimi, and T. L. Andresen, Mol. Ther., 21, 149 (2013).

(43) M. Creixell and N. A. Peppas, Nano Today, 7, 367 (2012).

(44) Y. Krishnamachari and A. K. Salem, Adv. Drug Deliv. Rev., 61, 205 (2009).

(45) H. Jung, S. A. Kim, E. Lee, and H. Mok, Macromol. Res., 23, 449 (2015).

(46) L. Kang, Z. Gao, W. Huang, M. Jin, and Q. Wang, Acta Pharm. Sin. B, 5, 169 (2015).

(47) A. M. Krieg, Nat. Rev. Drug Discov., 5, 471 (2006).

(48) N. Li, L. Zhao, L. Qi, Z. Li, and Y. Luan, Prog. Polym. Sci, 58, 1 (2016).

(49) Y. Wang, S. Gao, W.-H. Ye, H. S. Yoon, and Y.-Y. Yang, Nat. Mater., 5, 791 (2006).

(50) M. Sun, K. Wang, and D. Oupický, Adv. Healthc. Mater., 7, 1701070 (2018).

(51) Y. Gao, M. Wei, X. Li, W. Xu, A. Ahiabu, J. Perdiz, Z. Liu, and M. J. Serpe, Macromol. Res., 25, 513 (2017).

(52) L. E. Gerweck and K. Seetharaman, Cancer Res., 56, 1194 (1996).

(53) F. Wang, C.-H. Lu, and I. Willner, Chem. Rev., 114, 2881 (2014).

(54) B. Kang, S. V. Park, H. T. Soh, and S. S. Oh, ACS Sens., 4, 2802 (2019).

(55) M. Cho, S. Soo Oh, J. Nie, R. Stewart, M. Eisenstein, J. Chambers, J.D. Marth, F. Walker, J. A. Thomson, and H. T. Soh, Proc. Natl. Acad. Sci., 110, 18460 (2013).

(56) A. D. Keefe, S. Pai, and A. Ellington, Nat. Rev. Drug Discov., 9, 537 (2010).

(57) Y. Zhuang, H. Deng, Y. Su, L. He, R. Wang, G. Tong, D. He, and X. Zhu, Biomacromolecules, 17, 2050 (2016).

(58) A. Maruyama, Adv. Drug Deliv. Rev., 52, 227 (2001).

(59) M. Kwak and A. Herrmann, Angew. Chem. Int. Ed., 49, 8574 (2010).

(60) S. M. Hammond, A. Aartsma-Rus, S. Alves, S. E. Borgos, R. A. M. Buijsen, R. W. J. Collin, G. Covello, M. A. Denti, L. R. Desviat, L. Echevarría, C. Foged, G. Gaina, A. Garanto, A. T. Goyenvalle, M. Guzowska, I. Holod- 
nuka, D. R. Jones, S. Krause, T. Lehto, M. Montolio, W. Van Roon-Mom, and V. Arechavala-Gomeza, EMBO Mol. Med., 13 (2021).

(61) H. Yin, R. L. Kanasty, A. A. Eltoukhy, A. J. Vegas, J. R. Dorkin, and D. G. Anderson, Nat. Rev. Genet, 15, 541 (2014).

(62) U. Lächelt and E. Wagner, Chem. Rev., 115, 11043 (2015).

(63) S. F. Dowdy, Nat. Biotechnol., 35, 222 (2017).

(64) A. S. Piotrowski-Daspit, A. C. Kauffman, L. G. Bracaglia, and W. M. Saltzman, Adv. Drug Deliv. Rev., 156, 119 (2020).

(65) P. C. Zamecnik and M. L. Stephenson, Proc. Natl. Acad. Sci., 75, 280 (1978).

(66) J. K. Watts and D. R. Corey, J. Pathol., 226, 365 (2012).

(67) C. F. Bennett, Annu. Rev. Med., 70, 307 (2019).

(68) T. V. Chirila, P. E. Rakoczy, K. L. Garrett, X. Lou, and I. J. Constable, Biomaterials, 23, 321 (2002).

(69) R. L. Juliano, Nucleic Acids Res., 44, 6518 (2016).

(70) T. Merdan, J. KopečEk, and T. Kissel, Adv. Drug Deliv. Rev., 54, 715 (2002).

(71) E. Fattal and G. Barratt, Br. J. Pharmacol., 157, 179 (2009).

(72) G. De Rosa, F. Quaglia, A. Bochot, F. Ungaro, and E. Fattal, Biomacromolecules, 4, 529 (2003).

(73) S. Dheur, N. Dias, A. Van Aerschot, P. Herdewijn, T. Bettinger, J.-S. Remy, C. Hélène, and E. T. Saison-Behmoaras, Antisense Nucleic Acid Drug Dev., 9, 515 (1999).

(74) M. S. Falzarano, C. Passarelli, and A. Ferlini, Nucleic Acid Ther., 24, 87 (2014).

(75) S. Akhtar, M. D. Hughes, A. Khan, M. Bibby, M. Hussain, Q. Nawaz, J. Double, and P. Sayyed, Adv. Drug Deliv. Rev., 44, 3 (2000).

(76) R. A. Jain, Biomaterials, 21, 2475 (2000).

(77) K. J. Lewis, W. J. Irwin, and S. Akhtar, J. Drug Target, 5, 291 (1998).

(78) Y. Dorsett and T. Tuschl, Nat. Rev. Drug Discov., 3, 318 (2004).

(79) K. A. Whitehead, R. Langer, and D. G. Anderson, Nat. Rev. Drug Discov., 8, 129 (2009).

(80) J.-R. Bertrand, M. Pottier, A. Vekris, P. Opolon, A. Maksimenko, and C. Malvy, Biochem. Biophys. Res. Commun., 296, 1000 (2002).

(81) D. H. Kim and J. J. Rossi, Nat. Rev. Genet, 8, 173 (2007).

(82) K. Tatiparti, S. Sau, S. Kashaw, and A. Iyer, Nanomaterials, 7, 77 (2017).

(83) F. Alexis, E. Pridgen, L. K. Molnar, and O. C. Farokhzad, Mol. Pharm., 5, 505 (2008).

(84) S. Mao, M. Neu, O. Germershaus, O. Merkel, J. Sitterberg, U. Bakowsky, and T. Kissel, Bioconjug. Chem., 17, 1209 (2006).

(85) G. F. Walker, C. Fella, J. Pelisek, J. Fahrmeir, S. Boeckle, M. Ogris, and E. Wagner, Mol. Ther., 11, 418 (2005).

(86) J. Hoon Jeong, L. V. Christensen, J. W. Yockman, Z. Zhong, J. F. J. Engbersen, W. Jong Kim, J. Feijen, and S. Wan Kim, Biomaterials, 28, 1912 (2007).

(87) J. K. W. Lam, M. Y. T. Chow, Y. Zhang, and S. W. S. Leung, Mol. Ther. Nucleic Acids, 4, e252 (2015).

(88) R. C. Friedman, K. K. H. Farh, C. B. Burge, and D. P. Bartel, Genome Res., 19, 92 (2008).

(89) G. A. Calin, C. Sevignani, C. D. Dumitru, T. Hyslop, E. Noch, S. Yendamuri, M. Shimizu, S. Rattan, F. Bullrich, M. Negrini, and C. M. Croce, Proc. Natl. Acad. Sci., 101, 2999 (2004).

(90) H. Wang, Y. Jiang, H. Peng, Y. Chen, P. Zhu, and Y. Huang, Adv. Drug Deliv. Rev., 81, 142 (2015).

(91) A. F. Ibrahim, U. Weirauch, M. Thomas, A. Grünweller, R. K. Hartmann, and A. Aigner, Cancer Res., 71, 5214 (2011).

(92) P. S. Kowalski, A. Rudra, L. Miao, and D. G. Anderson, Mol. Ther., 27, 710 (2019).

(93) C. Lorenz, M. Fotin-Mleczek, G. Roth, C. Becker, T. C. Dam, W. P. R. Verdurmen, R. Brock, J. Probst, and T. Schlake, RNA Biol., 8, 627 (2011).

(94) C. Cheng, A. J. Convertine, P. S. Stayton, and J. D. Bryers, Biomaterials, 33, 6868 (2012).

(95) Y. Wang, Z. Zhang, J. Luo, X. Han, Y. Wei, and X. Wei, Mol. Cancer, 20
(2021).

(96) H. Yin, K. J. Kauffman, and D. G. Anderson, Nat. Rev. Drug Discov., 16, 387 (2017).

(97) U. Capasso Palmiero, J. C. Kaczmarek, O. S. Fenton, and D. G. Anderson, Adv. Healthc. Mater., 7, 1800249 (2018).

(98) R. A. Cordeiro, A. Serra, J. F. J. Coelho, and H. Faneca, J. Control. Release, 310, 155 (2019).

(99) J.S. Patton and P. R. Byron, Nat. Rev. Drug Discov., 6, 67 (2007).

(100) C. L. Densmore, F. M. Orson, B. Xu, B. M. Kinsey, J. C. Waldrep, P. Hua, B. Bhogal, and V. Knight, Mol. Ther., 1, 180 (2000).

(101) A.K. Patel, J.C. Kaczmarek, S. Bose, K.J. Kauffman, F. Mir, M.W. Heartlein, F. Derosa, R. Langer, and D.G. Anderson, Adv. Mater., 31, 1805116 (2019).

(102) Y. Jiang, A. Gaudin, J. Zhang, T. Agarwal, E. Song, A. C. Kauffman, G. T. Tietjen, Y. Wang, Z. Jiang, C. J. Cheng, and W. M. Saltzman, Biomaterials, 176, 122 (2018).

(103) J. Zhou, J. Liu, C. J. Cheng, T. R. Patel, C. E. Weller, J. M. Piepmeier, Z. Jiang, and W. M. Saltzman, Nat. Mater., 11, 82 (2012).

(104) Y. Li, J. Gao, C. Zhang, Z. Cao, D. Cheng, J. Liu, and X. Shuai, Top. Curr. Chem., 375, 27 (2017).

(105) T. Bus, A. Traeger, and U. S. Schubert, J. Mater. Chem. B, 6, 6904 (2018).

(106) H. Takemoto, K. Miyata, N. Nishiyama, and K. Kataoka, Adv. Genet, 88, 289 (2014).

(107) J. Liu, Y. Huang, A. Kumar, A. Tan, S. Jin, A. Mozhi, and X.-J. Liang, Biotechnol. Adv., 32, 693 (2014).

(108) Y.-B. Hu, E. B. Dammer, R.-J. Ren, and G. Wang, Transl. Neurodegener., 4 (2015).

(109) J. S. Suk, Q. Xu, N. Kim, J. Hanes, and L. M. Ensign, Adv. Drug Deliv. Rev., 99, 28 (2016).

(110) C.-F. Xu, H.-B. Zhang, C.-Y. Sun, Y. Liu, S. Shen, X.-Z. Yang, Y.-H. Zhu, and J. Wang, Biomaterials, 88, 48 (2016).

(111) I. M. S. Degors, C. Wang, Z. U. Rehman, and I. S. Zuhorn, Acc. Chem. Res., 52, 1750 (2019).

(112) K. Itaka, T. Ishii, Y. Hasegawa, and K. Kataoka, Biomaterials, 31, 3707 (2010).

(113) C. E. Nelson, A. J. Kim, E. J. Adolph, M. K. Gupta, F. Yu, K. M. Hocking, J. M. Davidson, S. A. Guelcher, and C. L. Duvall, Adv. Mater., 26, 607 (2014).

(114) P. Mi, Theranostics, 10, 4557 (2020).

(115) R. Cheng, F. Feng, F. Meng, C. Deng, J. Feijen, and Z. Zhong, J. Control. Release, 152, 2 (2011).

(116) Gerald and Tamas, Cell, 163, 560 (2015).

(117) Y. Chang, K. Yang, P. Wei, S. Huang, Y. Pei, W. Zhao, and Z. Pei, Angew. Chem. Int. Ed., 53, 13126 (2014).

(118) S. Ganta, H. Devalapally, A. Shahiwala, and M. Amiji,J. Control. Release, 126, 187 (2008).

(119) S. Choi and K.-D. Lee, J. Control. Release, 131, 70 (2008).

(120) N. W. S. Kam, Z. Liu, and H. Dai, J. Am. Chem. Soc., 127, 12492 (2005).

(121) S. Matsumoto, R. J. Christie, N. Nishiyama, K. Miyata, A. Ishii, M. Oba, H. Koyama, Y. Yamasaki, and K. Kataoka, Biomacromolecules, 10, 119 (2009).

(122) H.-L. Jiang, M. A. Islam, L. Xing, J. Firdous, W. Cao, Y.-J. He, Y.Zhu, K.-H. Cho, H.-S. Li, and C.-S. Cho, Top. Curr. Chem., 375, 34 (2017).

(123) Q. Peng, Z. Zhong, and R. Zhuo, Bioconjug. Chem., 19, 499 (2008).

(124) Y. Li, D. Maciel, J. Rodrigues, X. Shi, and H. Tomás, Chem. Rev., 115, 8564 (2015).

(125) K. Nam, J. W. Park, H. Bark, J. Han, J.-W. Nah, M.-K. Jang, and S. W. Kim, Macromol. Res., 22, 370 (2014).

(126) H. Kawagishi and T. Finkel, Nat. Med., 20, 711 (2014).

(127) M. Mittal, M. R. Siddiqui, K. Tran, S. P. Reddy, and A. B. Malik, Antioxid. Redox Signal., 20, 1126 (2014).

(128) J. R. Burgoyne, S.-I. Oka, N. Ale-Agha, and P. Eaton, Antioxid. Redox 
Signal., 18, 1042 (2013).

(129) M. S. Shim and Y. Xia, Angew. Chem. Int. Ed., 52, 6926 (2013).

(130) D. S. Wilson, G. Dalmasso, L. Wang, S. V. Sitaraman, D. Merlin, and N. Murthy, Nat. Mater., 9, 923 (2010).

(131) X. Xu, P. E. Saw, W. Tao, Y. Li, X. Ji, S. Bhasin, Y. Liu, D. Ayyash, J. Rasmussen, M. Huo, J. Shi, and O. C. Farokhzad, Adv. Mater., 29, 1700141 (2017).

(132) Y. Li, H. Bai, H. Wang, Y. Shen, G. Tang, and Y. Ping, Nanoscale, 10, 203 (2018).

(133) R. De La Rica, D. Aili, and M. M. Stevens, Adv. Drug Deliv. Rev., 64, 967 (2012).

(134) J. Hu, G. Zhang, and S. Liu, Chem. Soc. Rev., 41, 5933 (2012).

(135) P. Yingyuad, M. Mével, C. Prata, S. Furegati, C. Kontogiorgis, M. Thanou, and A. D. Miller, Bioconjug. Chem., 24, 343 (2013).

(136) C. Wang, Q. Chen, Z. Wang, and X. Zhang, Angew. Chem. Int. Ed., 49, 8612 (2010).

(137) Q. Hu, P. S. Katti, and Z. Gu, Nanoscale, 6, 12273 (2014).

(138) H.-X. Wang, X.-Z. Yang, C.-Y. Sun, C.-Q. Mao, Y.-H. Zhu, and J. Wang, Biomaterials, 35, 7622 (2014).

(139) G. Bergers, R. Brekken, G. McMahon, T. H. Vu, T. Itoh, K. Tamaki, K. Tanzawa, P. Thorpe, S. Itohara, Z. Werb, and D. Hanahan, Nat. Cell Biol., 2, 737 (2000).

(140) V. W. Yong, C. Power, P. Forsyth, and D. R. Edwards, Nat. Rev. Neurosci., 2, 502 (2001).

(141) N. Qiu, X. Liu, Y. Zhong, Z. Zhou, Y. Piao, L. Miao, Q. Zhang, J. Tang, L. Huang, and Y. Shen, Adv. Mater., 28, 10613 (2016).

(142) A. Raza, U. Hayat, T. Rasheed, M. Bilal, and H. M. N. Iqbal,J. Mater. Res. Technol., 8, 1497 (2019).

(143) K. Kono, T. Ozawa, T. Yoshida, F. Ozaki, Y. Ishizaka, K. Maruyama, C. Kojima, A. Harada, and S. Aoshima, Biomaterials, 31, 7096 (2010).

(144) T. Karino, S. Koga, and M. Maeta, Jpn. J. Surg., 18, 276 (1988).

(145) C. W. Song, H. J. Park, C. K. Lee, and R. Griffin, Int. J. Hyperth, 21, 761 (2005).

(146) G. Kong, G. Anyarambhatla, W. P. Petros, R. D. Braun, O. M. Colvin, D. Needham, and M. W. Dewhirst, Cancer Res., 60, 6950 (2000).

(147) L. H. Lindner, M. E. Eichhorn, H. Eibl, N. Teichert, M. Schmitt-Sody, R. D. Issels, and M. Dellian, Clin. Cancer Res., 10, 2168 (2004).

(148) B. R. Twaites, C. De Las Heras Alarcón, M. Lavigne, A. Saulnier, S. S. Pennadam, D. Cunliffe, D. C. Górecki, and C. Alexander, J. Control. Release, 108, 472 (2005).

(149) D. Roy, W. L. A. Brooks, and B. S. Sumerlin, Chem. Soc. Rev., 42, 7214 (2013).

(150) G. Pasparakis and C. Tsitsilianis, Polymer, 211, 123146 (2020).

(151) M. A. Haq, Y. Su, and D. Wang, Mater. Sci. Eng. C, 70, 842 (2017).

(152) J.-W. Seo and U. S. Shin, Macromol. Res., 23, 1073 (2015).

(153) A. Zintchenko, M. Ogris, and E. Wagner, Bioconjug. Chem., 17, 766 (2006).

(154) A. Schwerdt, A. Zintchenko, M. Concia, N. Roesen, K. Fisher, L. H. Lindner, R. Issels, E. Wagner, and M. Ogris, Hum. Gene Ther., 19, 1283 (2008).

(155) P. Cao, X. Sun, Y. Liang, X. Gao, X. Li, W. Li, Z. Song, W. Li, and G. Liang, Nanomedicine, 10, 1585 (2015).

(156) S. Hernot and A. L. Klibanov, Adv. Drug Deliv. Rev., 60, 1153 (2008).

(157) K. Ferrara, R. Pollard, and M. Borden, Annu. Rev. Biomed. Eng., 9, 415 (2007).

(158) G. Shapiro, A. W. Wong, M. Bez, F. Yang, S. Tam, L. Even, D. Sheyn, S. Ben-David, W. Tawackoli, G. Pelled, K. W. Ferrara, and D. Gazit, J. Control. Release, 223, 157 (2016).

(159) F. Meng, Z. Zhong, and J. Feijen, Biomacromolecules, 10, 197 (2009).

(160) N. D. Yan, W. Keller, N. M. Scully, D. R. S. Lean, and P. J. Dillon, Nature, 381, 141 (1996).
(161) C. Ash, M. Dubec, K. Donne, and T. Bashford, Lasers Med. Sci, 32, 1909 (2017).

(162) G. Liu, W. Liu, and C.-M. Dong, Polym. Chem., 4, 3431 (2013).

(163) J. Shin, J. Sung, M. Kang, X. Xie, B. Lee, K. M. Lee, T. J. White, C. Leal, N. R. Sottos, P. V. Braun, and D. G. Cahill, Proc. Natl. Acad. Sci., 116, 5973 (2019).

(164) J. Y. Park, M. Umashankar, and D. S. Huh, Macromol. Res., 24, 350 (2016).

(165) Y. Li, J. Yang, L. Sun, W. Wang, and W. Liu, J. Mater. Chem. B, 2, 3868 (2014).

(166) S. Duan, D. Cao, X. Li, H. Zhu, M. Lan, Z. Tan, Z. Song, R. Zhu, L. Yin, and Y. Chen, Biomaterials Science, 8, 290 (2020).

(167) X. Deng, N. Zheng, Z. Song, L. Yin, and J. Cheng, Biomaterials, 35, 5006 (2014).

(168) S. K. M. Nalluri, J. Voskuhl, J. B. Bultema, E. J. Boekema, and B. J. Ravoo, Angew. Chem. Int. Ed., 50, 9747 (2011).

(169) M. S. Shim and Y. J. Kwon, Adv. Drug Deliv. Rev., 64, 1046 (2012).

(170) V. I. Shubayev, T. R. Pisanic, and S. Jin, Adv. Drug Deliv. Rev., 61, 467 (2009).

(171) Y.-L. Lo, H.-L. Chou, Z.-X. Liao, S.-J. Huang, J.-H. Ke, Y.-S. Liu, C.-C. Chiu, and L.-F. Wang, Nanoscale, 7, 8554 (2015).

(172) X. Han, K. Xu, O. Taratula, and K. Farsad, Nanoscale, 11, 799 (2019).

(173) T. Park, J. Jeong, and S. Kim, Adv. Drug Deliv. Rev., 58, 467 (2006).

(174) L. Brannon-Peppas and J.O. Blanchette, Adv. Drug Deliv. Rev., 56, 1649 (2004).

(175) S.-J. Chiu, N. T. Ueno, and R. J. Lee, J. Control. Release, 97, 357 (2004).

(176) A. Mitra, J. Mulholland, A. Nan, E. McNeill, H. Ghandehari, and B. R. Line, J. Control. Release, 102, 191 (2005).

(177) L. Yang, H. Sun, Y. Liu, W. Hou, Y. Yang, R. Cai, C. Cui, P. Zhang, X. Pan, X. Li, L. Li, B. S. Sumerlin, and W. Tan, Angew. Chem., 130, 17294 (2018).

(178) S. Mignani, X. Shi, V. Ceña, and J.-P. Majoral, Drug Discov. Today, 25, 1065 (2020).

(179) S. S. Oh, B. F. Lee, F. A. Leibfarth, M. Eisenstein, M. J. Robb, N. A. Lynd, C. J. Hawker, and H. T. Soh, J. Am. Chem. Soc., 136, 15010 (2014).

(180) M. Alibolandi, S. M. Taghdisi, P. Ramezani, F. Hosseini Shamili, S. A. Farzad, K. Abnous, and M. Ramezani, Int. J. Pharm., 519, 352 (2017).

(181) M. F. Sanmamed and L. Chen, Cell, 175, 313 (2018).

(182) L. Xing, T.-J. Zhou, Y.-T. Fan, Y.-J. He, T. Pang, K.-H. Cho, J.-J. Lu, H.-L. Jiang, and C.-S. Cho, Macromol. Res., 27, 215 (2019).

(183) C. H. June, R. S. O'Connor, O. U. Kawalekar, S. Ghassemi, and M. C. Milone, Science, 359, 1361 (2018).

(184) J. Vollmer and A. M. Krieg, Adv. Drug Deliv. Rev., 61, 195 (2009).

(185) M. Kumar, B. Keller, N. Makalou, and R. E. Sutton, Hum. Gene Ther., 12, 1893 (2001).

(186) B. R. Olden, Y. Cheng, J. L. Yu, and S. H. Pun, J. Control. Release, 282, 140 (2018).

(187) P. Sharma, S. Hu-Lieskovan, J. A. Wargo, and A. Ribas, Cell, 168, 707 (2017).

(188) C. H. June and M. Sadelain, N. Engl. J. Med., 379, 64 (2018).

(189) J. T. Wilson, S. Keller, M. J. Manganiello, C. Cheng, C.-C. Lee, C. Opara, A. Convertine, and P. S. Stayton, ACS Nano, 7, 3912 (2013).

(190) A. Kundu, S. Nandi, and A. K. Nandi, Prog. Mater Sci, 88, 136 (2017).

(191) Y. Mi, C. T. Hagan, B. G. Vincent, and A. Z. Wang, Adv. Sci., 6, 1801847 (2019).

(192) H. Lv, S. Zhang, B. Wang, S. Cui, and J. Yan, J. Control. Release, 114, 100 (2006).

(193) R. Duncan and M. J. Vicent, Adv. Drug Deliv. Rev., 65, 60 (2013).

Publisher's Note Springer Nature remains neutral with regard to jurisdictional claims in published maps and institutional affiliations. 